\title{
HUBBLE SPACE TELESCOPE/ADVANCED CAMERA FOR SURVEYS MORPHOLOGY OF Ly $\alpha$ EMITTERS AT REDSHIFT 5.7 IN THE COSMOS FIELD*
}

\author{
Y. Taniguchi ${ }^{1}$, T. Murayama ${ }^{2}$, N. Z. Scoville ${ }^{3}$, S. S. Sasaki ${ }^{2,4}$, T. NagaO ${ }^{1,4}$, Y. Shioya ${ }^{1}$, T. Saito ${ }^{1}$, Y. Ideue ${ }^{4}$, \\ A. Nakajima ${ }^{4}$, K. Matsuoka ${ }^{4}$, D. B. Sanders ${ }^{5}$, B. Mobasher ${ }^{6}$, H. Aussel ${ }^{7}$, P. Capak ${ }^{3,8}$, M. Salvato $^{3}$, A. Koekemoer ${ }^{9}$, \\ C. Carilli $^{10}$, A. Cimatti ${ }^{11}$, R. S. Ellis ${ }^{3}$, B. Garilli ${ }^{12}$, M. Giavalisco $^{13}$, O. IlberT ${ }^{5}$, C. D. ImPey ${ }^{14}$, M. G. KitzbichleR ${ }^{15}$, O. Le \\ Fevre $^{16}$, H. J. McCracken ${ }^{17}$, C. Scarlata ${ }^{3}$, E. Schinnerer ${ }^{18}$, V. Smolcic ${ }^{3}$, S. Tribiano ${ }^{19}$, And J. R. Trump ${ }^{14}$ \\ ${ }^{1}$ Research Center for Space and Cosmic Evolution, Ehime University, Bunkyo-cho 2-5, Matsuyama 790-8577, Japan \\ ${ }^{2}$ Astronomical Institute, Graduate School of Science, Tohoku University, Aramaki, Aoba, Sendai 980-8578, Japan \\ ${ }^{3}$ Department of Astronomy, MS 105-24, California Institute of Technology, Pasadena, CA 91125, USA \\ ${ }^{4}$ Graduate School of Science and Engineering, Ehime University, Bunkyo-cho, Matsuyama 790-8577, Japan \\ ${ }^{5}$ Institute for Astronomy, University of Hawaii, 2680 Woodlawn Drive, HI 96822, USA \\ ${ }^{6}$ Department of Physics and Astronomy, University of California, Riverside, CA 92521, USA \\ ${ }^{7}$ CEA/DSM-CNRS, Université Paris Diderot, DAPNIA/SAp, Orme des Merisiers, 91191 Gif-sur-Yvette, France \\ ${ }^{8}$ Spitzer Science Center, California Institute of Technology, Pasadena, CA 91125, USA \\ ${ }^{9}$ Space Telescope Science Institute, 3700 San Martin Drive, Baltimore, MD 21218, USA \\ ${ }^{10}$ National Radio Astronomy Observatory, P.O. Box 0, Socorro, NM 87801-0387, USA \\ ${ }^{11}$ Dipartamento di Astronmia, Universita di Bologna, Italy \\ ${ }^{12}$ INAF, Istituto di Astrofisica Spaziale e Fisica Cosmica, Sezione di Milano, via Bassini 15, 20133 Milano, Italy \\ ${ }^{13}$ Department of Astronomy, University of Massachusetts, Amherst, MA 01003, USA \\ ${ }^{14}$ Steward Observatory, University of Arizona, Tucson, AZ 85721, USA \\ ${ }^{15}$ Max-Planck-Institut für Astrophysik, D-85748 Garching bei München, Germany \\ ${ }^{16}$ Laboratoire d'Astrophysique de Marseille, BP 8, Traverse du Siphon, 13376 Marseille Cedex 12, France \\ ${ }^{17}$ Institut d'Astrophysique de Paris, 98 bis Boulevard Arago, F-75014 Paris, France \\ ${ }^{18}$ Max Planck Institut für Astronomie, Königstuhl 17, Heidelberg D-69117, Germany \\ ${ }^{19}$ City University of New York, Borough of Manhatan Community College, 199 Chambers Street, New York, NY 10007, USA \\ Received 2008 November 3; accepted 2009 June 15; published 2009 July 28
}

\begin{abstract}
We present detailed morphological properties of Ly $\alpha$ emitters (LAEs) at $z \approx 5.7$ in the COSMOS field based on Hubble Space Telescope Advanced Camera for Surveys (ACS) data. The ACS imaging in the F814W filter covered 85 LAEs of the 119 LAEs identified in the full two square degree field, and 47 LAEs of them are detected in the ACS images. Nearly half of them are spatially extended with a size larger than $0.15 \operatorname{arcsec}(\sim 0.88 \mathrm{kpc}$ at $z=5.7)$ and up to $0.4 \operatorname{arcsec}(\sim 2.5 \mathrm{kpc}$ at $z=5.7)$. The others are nearly unresolved compact objects. Two LAEs show double-component structures indicating interaction or merging of building components to form more massive galaxies. By stacking the ACS images of all the detected sources, we obtain a Sersic parameter of $n \sim 0.7$ with a half-light radius of $0.13 \operatorname{arcsec}(0.76 \mathrm{kpc})$, suggesting that the majority of ACS detected LAEs have not spheroidal-like but disk-like or irregular light profiles. Comparing ACS F814W magnitudes $\left(I_{814}\right)$ with Subaru/Suprime-Cam magnitudes in the NB816, $i^{\prime}$, and $z^{\prime}$ bands, we find that the ACS imaging in the F814W band mainly probes UV continuum rather than Ly $\alpha$ line emission. UV continuum sizes tend to be larger for LAEs with larger Ly $\alpha$ emission regions as traced by the NB816 imaging. The nondetection of 38 LAEs in the ACS images is likely due to the fact that their surface brightness is too low both in the UV continuum and Ly $\alpha$ emission. Estimating $I_{814}$ for the ACS-undetected LAEs from the $z^{\prime}$ and NB816 magnitudes, we find that 16 of these are probably LAEs with a size larger than 0.15 arcsec in UV continuum. All these results suggest that our LAE sample contains systematically larger LAEs in UV continuum size than those previously studied at $z \sim 6$.
\end{abstract}

Key words: cosmology: observations - early universe - galaxies: evolution - galaxies: formation - galaxies: high-redshift

\section{INTRODUCTION}

During the last decade, a large number of young, star-forming galaxies beyond redshift of $z=5$ have been found based on deep imaging observations of both the Hubble Space Telescope $(H S T)$ and $8-10 \mathrm{~m}$ class telescopes such as the $8.2 \mathrm{~m}$ Subaru Telescope (see for a review, Bouwens \& Illingworth 2006; Taniguchi 2008; see also for recent progress, Bouwens et al. 2008, 2009; Bradley et al. 2008). In particular, narrowband

\footnotetext{
* Based on observations with NASA/ESA Hubble Space Telescope, obtained at the Space Telescope Science Institute, which is operated by AURA, Inc., under NASA contract NAS 5-26555; and also based on data collected at Subaru Telescope, which is operated by the National Astronomical Observatory of Japan.
}

imaging surveys have been providing us well defined samples of strong Ly $\alpha$ emitters (LAEs) at $z \approx 5.7$ (e.g., Rhoads \& Malhotra 2001; Ajiki et al. 2003; Hu et al. 2004; Shimasaku et al. 2006; Murayama et al. 2007 and references therein). These surveys are used to investigate the star formation activity in such young galaxies, providing typical star formation rates from several to a few tens $M_{\odot} \mathrm{yr}^{-1}$. Clustering properties are also one of the important issues studied as structure formation in the early universe provides an important observational constraint on hierarchical structure formation scenarios (e.g., Springel et al. 2005). Although a possible overdensity region has been identified in the Subaru XMM-Newton Deep Survey Field (Ouchi et al. 2005), there is no other significant evidence for clustering of young galaxies at $z \approx 5.7$ in other deep 
wide-field surveys (e.g., Shimasaku et al. 2006; Murayama et al. 2007).

Another interesting question is addressed to morphological properties of LAEs because these properties give us insights on how LAEs were assembled and how their intense star formation events were triggered. However, no systematic investigation of the detailed morphology of high- $z$ LAEs has yet been undertaken, although some case studies have been reported (e.g., Rhoads et al. 2005; Venemans et al. 2005; Pizkal et al. 2007; Overzier et al. 2008 and references therein). Rhoads et al. (2005) found in the Hubble Ultra Deep Field (UDF) a LAE at $z=5.4$ (UDF 5225) with a spatial extent of $0.25 \operatorname{arcsec} \times 1.0 \operatorname{arcsec}$ $(1.6 \mathrm{kpc} \times 6.3 \mathrm{kpc})$. This LAE shows a core together with three knots that appear to emanate from the core. On the other hand, Bunker et al. (2003) found a very compact LAE at $z=5.78$ in the Chandra Deep Field South among their $i$-dropout sample. Its half-light radius is only 0.08 arcsec (490 pc), barely resolved by the ACS PSF (0.06 arcsec). Stanway et al. (2004a) found three LAEs in the UDF during the course of their GLARE (Gemini Ly $\alpha$ at Reionization Era) project and obtained also small sizes ranging from $0.09 \operatorname{arcsec}(\sim 500 \mathrm{pc})$ to $0.14 \operatorname{arcsec}(\sim 900 \mathrm{pc})$. Relative small sizes of six LAEs with respect to Lyman break galaxies (LBGs) at similar redshift $(z \sim 6)$ are also found by Dow-Hygelund et al. (2007). Since the size and morphological properties provide us with useful insight on the understanding of physical processes of star formation, we need systematic studies of the detailed morphological properties for a large sample of such high- $z$ galaxies.

Recently, Murayama et al. (2007; hereafter M07) identified a total of 119 LAEs at $z \approx 5.7$ in the COSMOS field (Scoville et al. 2007a), providing one of the largest samples of LAEs in a large contiguous field. Since F814W imaging taken with the Advanced Camera for Surveys (ACS) on board the HST is available for the COSMOS field (Scoville et al. 2007b; Koekemoer et al. 2007), the sizes and morphologies of the LAEs in the COSMOS field can be investigated in detail. In particular, since the redshifted Ly $\alpha$ emission of our LAE sample is probed in the $\mathrm{F} 814 \mathrm{~W}$ imaging, it is possible to study the Ly $\alpha$ morphology as well as the rest-frame ultraviolet continuum shape. In this paper, we present our detailed analysis of ACS images of the LAE sample of M07.

We use a standard cosmology with $\Omega_{\text {matter }}=0.3, \Omega_{\Lambda}=0.7$, and $H_{0}=70 \mathrm{~km} \mathrm{~s}^{-1} \mathrm{Mpc}^{-1}$. Throughout this paper, we use magnitudes in the $\mathrm{AB}$ system.

\section{OBSERVATIONAL DATA AND ACS COUNTERPARTS OF LAEs}

In M07, $119 \mathrm{LAE}$ candidates at $5.65<z<5.75$ were carefully selected from optical imaging with both the narrowband filter, NB816 $\left(\lambda_{c}=8150 \AA\right.$ with a width $\Delta \lambda=120 \AA$; see Ajiki et al. 2003 for details) and broadband filters from $B$ to $z^{\prime}$ taken for a $1.95 \mathrm{deg}^{2}$ area of the COSMOS field using SuprimeCam (Miyazaki et al. 2002) on the Subaru Telescope (Kaifu et al. 2000; Iye et al. 2004). Details of the Subaru observations and data processing are described by Taniguchi et al. (2007) and Capak et al. (2007). Note that follow-up spectroscopy has been performed for 24 LAEs in the sample and that all of them showed Ly $\alpha$ emission at $z \approx 5.7$ (P. Capak et al. 2009, in preparation) verifying the effectiveness of the adopted selection method.

The ACS data in the F814W filter were taken for an area of $1.64 \mathrm{deg}^{2}$ of the COSMOS field and were processed to 0 . 05 pixel $^{-1}$ images with an averaged point-spread function

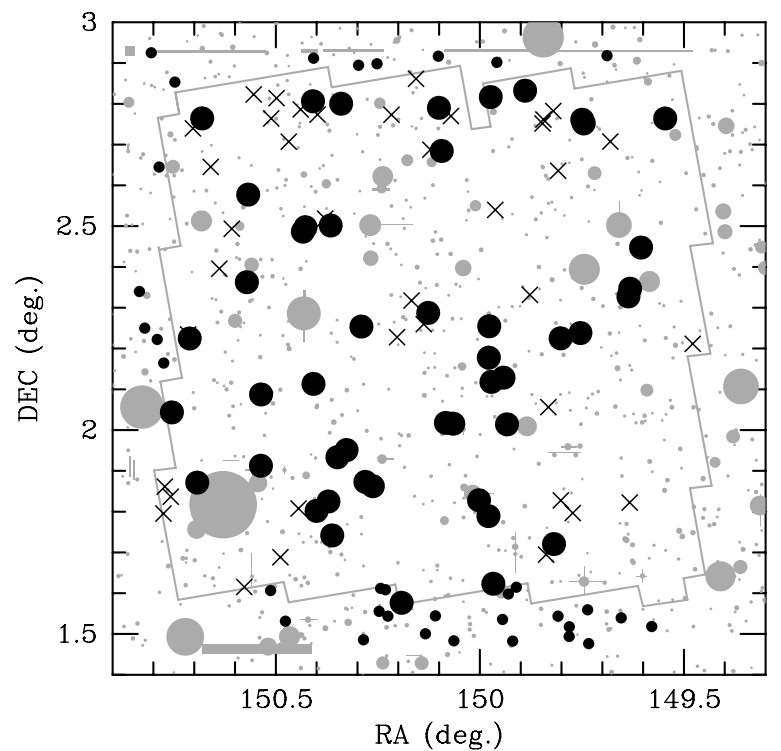

Figure 1. Spatial distribution of our sample of 119 LAEs (M07). The whole area is $1.95 \mathrm{deg}^{2}$ that is mapped with our Suprime-Cam observations. The ACS image is available for $1.64 \mathrm{deg}^{2}$, its footprint is indicated by the solid gray line. Masked out regions are shown by filled gray circles or thin gray lines. The 47 LAEs detected with ACS are shown by large filled circles while 38 LAEs undetected in the ACS images are shown by crosses. The remaining 34 LAEs shown by dots fall outside the HST/ACS field.

(PSF) width of 0.'097 (Scoville et al. 2007b; Koekemoer et al. 2007). In our analysis, we use the official COSMOS ACS image, Version 1.3.

Since the observed area of the ACS imaging is slightly smaller than that of the Subaru, we find that ACS data are available for 85 LAEs in the LAE sample defined by M07. Spatial distribution of these LAEs is shown with red symbols in Figure 1. Among them, 20 LAEs are already spectroscopically identified as star-forming galaxies at $z \sim 5.7$ (P. Capak et al. 2009, in preparation).

Our data analysis procedures for ACS data are as follow. First, for each LAE, we created a small ACS cutout $\left(16^{\prime \prime} \times 16^{\prime \prime}\right)$ centered on the LAE position (as derived in the NB816 image; see M07). Then, we used SExtractor (Bertin \& Arnouts 1996) to detect a possible ACS counterpart of the LAE. We adopted the following SExtractor parameters: a detection threshold of $1.6 \sigma$ and a minimum detection area of 9 pixels. These values were carefully selected (1) to allow for the detection both diffuse elongated sources as well as point-like sources, (2) to minimize false detections, and (3) to minimize detection failures for sources that are apparently shown in the ACS image by eyes. A Gaussian-profile filter ( 5 pixels $\times 5$ pixels $)$ with an FWHM of 2 pixels (0!. 1$)$ well matched to the PSF size was applied to the detection images for smoothing. The SExtractor parameters for the source detection in $I_{814}$ are listed in Table 1 .

We found 58 sources detected near the LAE positions $(r \leqslant$ $\left.1^{\prime \prime}\right)$. By eye inspection we rejected three sources because they are largely offset $(\geqslant 0$ '. 68$)$ from the LAE center and have no distinct counterpart in the Subaru broadband images of any bands. Next, in order to remove low- $z$ foreground neighbors, we examined the Subaru $B, V$, and $g^{\prime}$ images. Since the observed wavelength of the Lyman limit at $z \approx 5.7$ shifts to $6110 \AA$, a true LAE must be undetectable in these bands. In this analysis, we adopted the search radius for ACS counterparts is $1^{\prime \prime}$ and then removed six sources. 
Table 1

SExtractor Parameters for Source Detection

\begin{tabular}{|c|c|c|}
\hline Parameter & Value & Comment \\
\hline DETECT_TYPE & $\mathrm{CCD}$ & Detector type \\
\hline DETECT_MINAREA & 9 & Minimum number of pixels above threshold \\
\hline DETECT_THRESH & 1.6 & Detection threshold in sigma \\
\hline ANALYSIS_THRESH & 2.0 & Limit for isophotal analysis in sigma \\
\hline FILTER & $\mathrm{Y}$ & Apply filter for detection \\
\hline FILTER_NAME & gauss_2.0_5x5.conv & Name of the filter for detection \\
\hline DEBLEND_NTHRESH & 32 & Number of deblending subthresholds \\
\hline DEBLEND_MINCONT & 0.015 & Minimum contrast parameter for deblending \\
\hline CLEAN & $\mathrm{Y}$ & Clean spurious detection \\
\hline CLEAN_PARAM & 1.0 & Cleaning efficiency \\
\hline MASK_TYPE & CORRECT & Correct flux for blended source \\
\hline PHOT_AUTOPARAMS & $2.5,0.5$ & MAG_AUTO parameters: Kron factor and minimum radius \\
\hline SATUR_LEVEL & 50,000 & Level (in ADUs) at which saturation arises \\
\hline MAG_ZEROPOINT & 25.936 & Magnitude zero point \\
\hline MAG_GAMMA & 4.0 & Gamma of emulsion (for photographic scans) \\
\hline GAIN & 1.0 & Detector gain in $\mathrm{e}^{-} / \mathrm{ADU}$ \\
\hline PIXEL_SCALE & 0 & Size of pixel in $\operatorname{arcsec}(0=$ use FITS WCS info $)$ \\
\hline SEEING_FWHM & 0.11 & Stellar FWHM in arcsec \\
\hline BACK_SIZE & 64 & Background mesh size \\
\hline BACK_FILTERSIZE & 3 & Background filter size \\
\hline BACKPHOTO_TYPE & GLOBAL & Photometry background subtraction type \\
\hline
\end{tabular}

Table 2

COSMOS $z \approx 5.7$ LAE Sample

\begin{tabular}{lcc}
\hline \hline \multicolumn{1}{c}{ LAE Sample } & Number of LAEs & $\begin{array}{c}\text { Spectroscopic } \\
\text { Confirmation }\end{array}$ \\
\hline Total (Murayama et al. 2007) & 119 & 24 \\
Out of the ACS/F814W field & 34 & 4 \\
In the ACS/F814W field & 85 & 20 \\
ACS/F814W undetected & 38 & 3 \\
ACS/F814W detected & 47 & 17 \\
Double & 2 & 2 \\
\hline
\end{tabular}

Finally, among the 85 LAEs whose ACS data are available, we found 49 ACS sources for 47 LAEs $(17$ objects were spectroscopically confirmed; P. Capak et al. 2009, in preparation). Two LAEs (Nos 60 and 110) have double-component ACS sources. Offsets of the ACS positions from the NB816 positions are typically less than $0^{\prime \prime} 13$ in the ACS images, smaller than the pixel scale $\left(00^{\prime} 15\right.$ pixel $\left.^{-1}\right)$ of the NB816 images. The remaining 38 LAEs (three objects were spectroscopically confirmed; P. Capak et al. 2009, in preparation) are not detected in the ACS images. The numbers of the total sample, both the detected and undetected LAEs are summarized in Table 2.

In Figure 2, we show thumbnails of the 85 LAEs in the ACS F814W images. We also show thumbnails in the ACS F814W images together with their Subaru $i^{\prime}$, NB816, and $z^{\prime}$ images in Figure 3. Smoothed F814W images with a gaussian kernel with an FWHM of 2 pixels are also presented. The detected sources identified as LAE counterparts are indicated by red ellipses on the smoothed ACS, NB816, and $z^{\prime}$ images. Green and blue ellipses are ACS sources excluded from the sample by eye inspection and by rejection of foreground neighbors, respectively.

As shown in Figure 1, the ACS-detected (large filled circles) and ACS-undetected LAEs (crosses) appear to be almost randomly distributed in the COSMOS field, and thus their distributions may not be affected by large-scale inhomogeneity of the ACS data quality (e.g., edges of the field). The total magnitude $\left(I_{814}\right)$ and half-light radius $\left(R_{\mathrm{HL}}\right)$ were measured for each de- tected source by SExtractor on the original ACS image (i.e., not on the smoothed image). We adopted SExtractor's MAG_AUTO as total magnitude. For the 38 LAEs undetected in the F814W image, we estimated $3 \sigma$ upper limits for the magnitudes within a $1^{\prime \prime}$ diameter aperture. These photometric properties of the ACS data are listed in Table 3 . Note that the $3 \sigma$ limiting magnitude of the F814W images is $27.3 \mathrm{mag}$ in a $1^{\prime \prime}$ diameter aperture. All magnitudes are corrected for the Galactic extinction of $A_{\mathrm{F} 814 \mathrm{~W}}$ $=0.035$ (Capak et al. 2007). In Table 4, we list the photometric properties of the LAE candidates from M07. The $3 \sigma$ limiting magnitudes within a $2^{\prime \prime}$ diameter aperture in the NB816, $i^{\prime}$, and $z^{\prime}$ images are 25.7, 26.1, and 25.3, respectively.

\section{MORPHOLOGICAL PROPERTIES}

\subsection{Half-Light Radius}

The ACS counterparts of the LAEs look differently from object to object as shown in Figure 2. Some LAEs show compact, round, and nearly unresolved shapes, while others have an elongated, filamentary, or patchy morphology.

First, we analyze the sizes of our LAE sample in the ACS images. We show the distribution of half-light radius $\left(R_{\mathrm{HL}}\right)$ for the 47 ACS-detected LAEs in Figure 4. In this histogram, the size of the larger component is adopted for the two LAEs with double-component ACS sources. Note that the measured halflight radii of stars in the ACS images are typically $00^{\prime} 11$ as indicated in Figure 4. The number of LAEs in each $R_{\mathrm{HL}}$ bin decreases with increasing half-light radius beyond $0^{\prime \prime} 15$ while the overall distribution appears to be almost flat at the radius of $R_{\mathrm{HL}}<0$ '.'15. Therefore, we may conclude that LAEs with $R_{\mathrm{HL}}<0^{\prime \prime} 15$ are almost unresolved compact objects. Three LAEs (Nos. 40, 68, and 78) have a larger size as $R_{\mathrm{HL}} \geqslant 0$ 0!3 compared with the PSF size. However, the sizes of all the LAEs detected in our ACS imaging are smaller than 0.'4, and no widely extended LAE (like the LAE found by Rhoads et al. 2005) has been found.

In Figure 5, we show the distribution of $I_{814}$. This distribution is affected by detection incompleteness toward fainter magnitudes because the detection is limited by surface brightness. For 

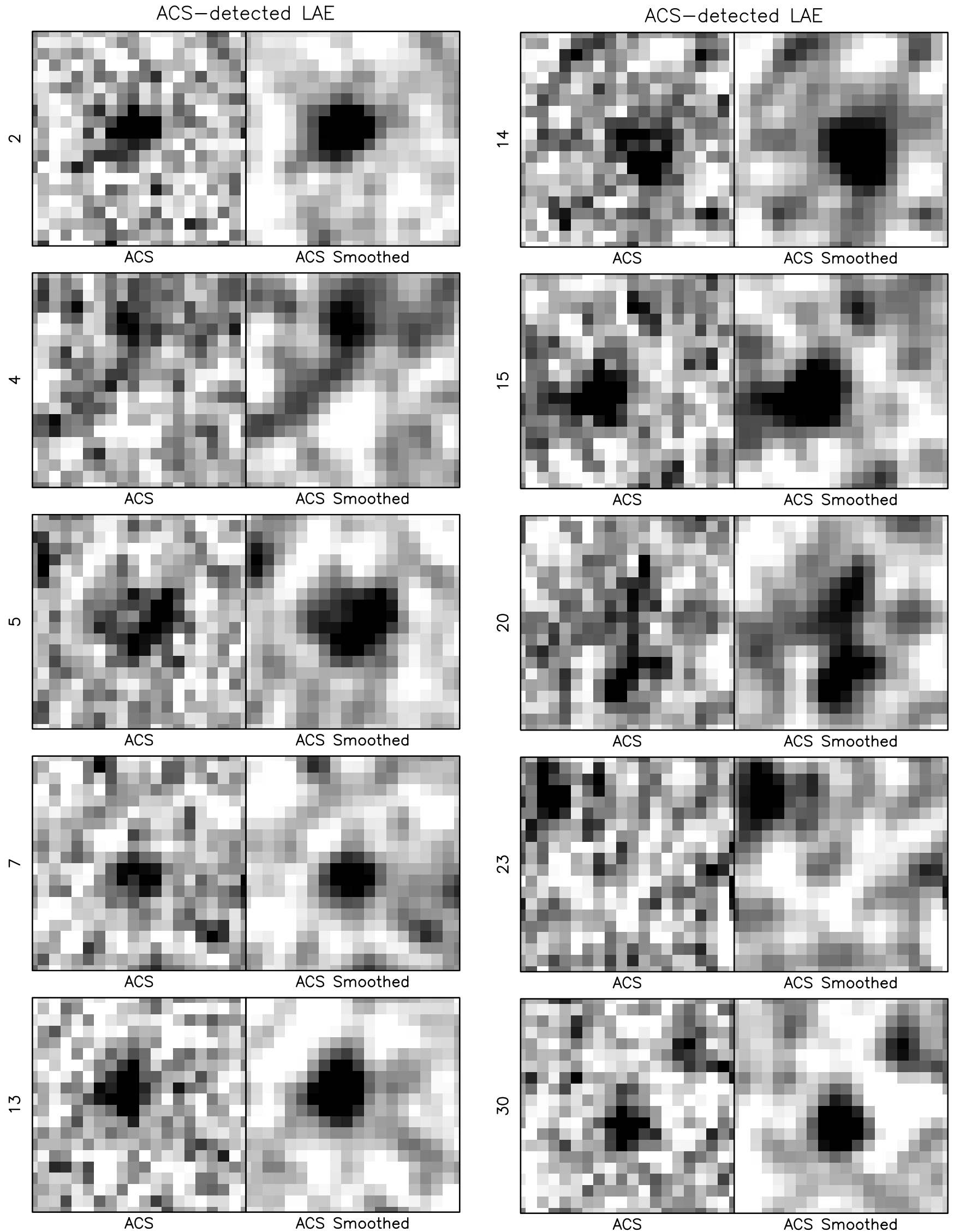

Figure 2. Thumbnails of all 85 LAEs analyzed in this paper. Each panel has a size of $1^{\prime \prime} \times 1^{\prime \prime}$. North is up and east is left. 

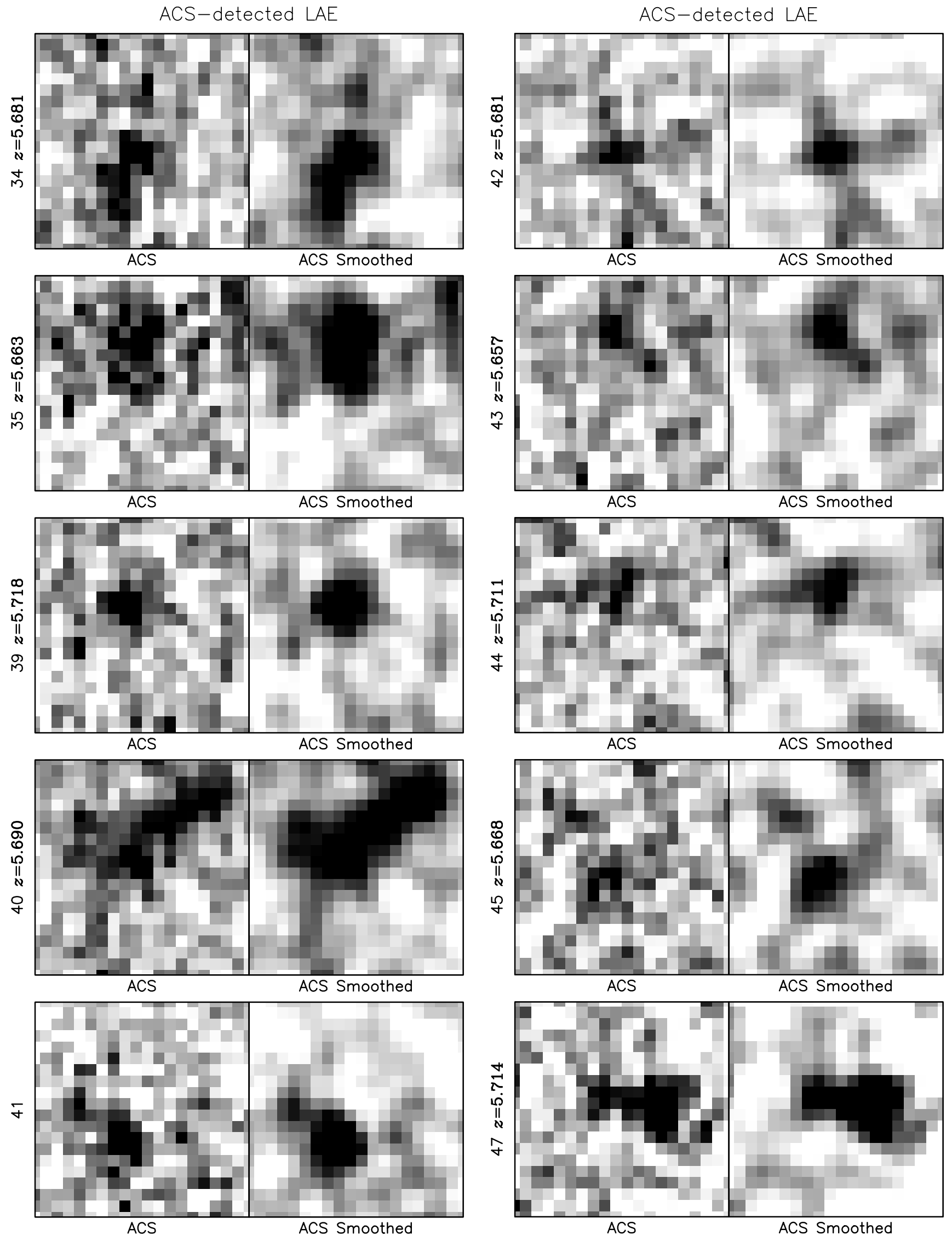

Figure 2. (Continued) 

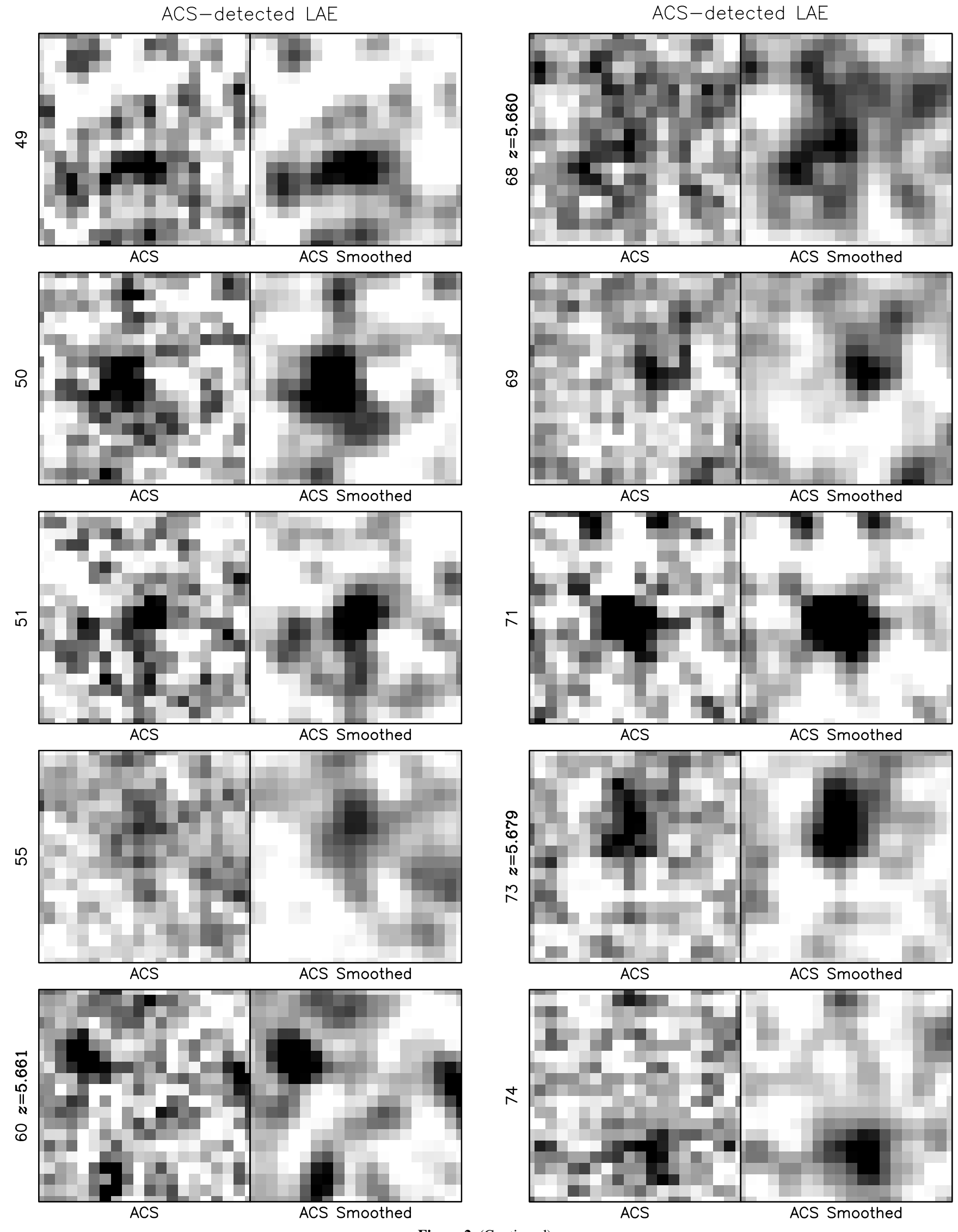

Figure 2. (Continued) 

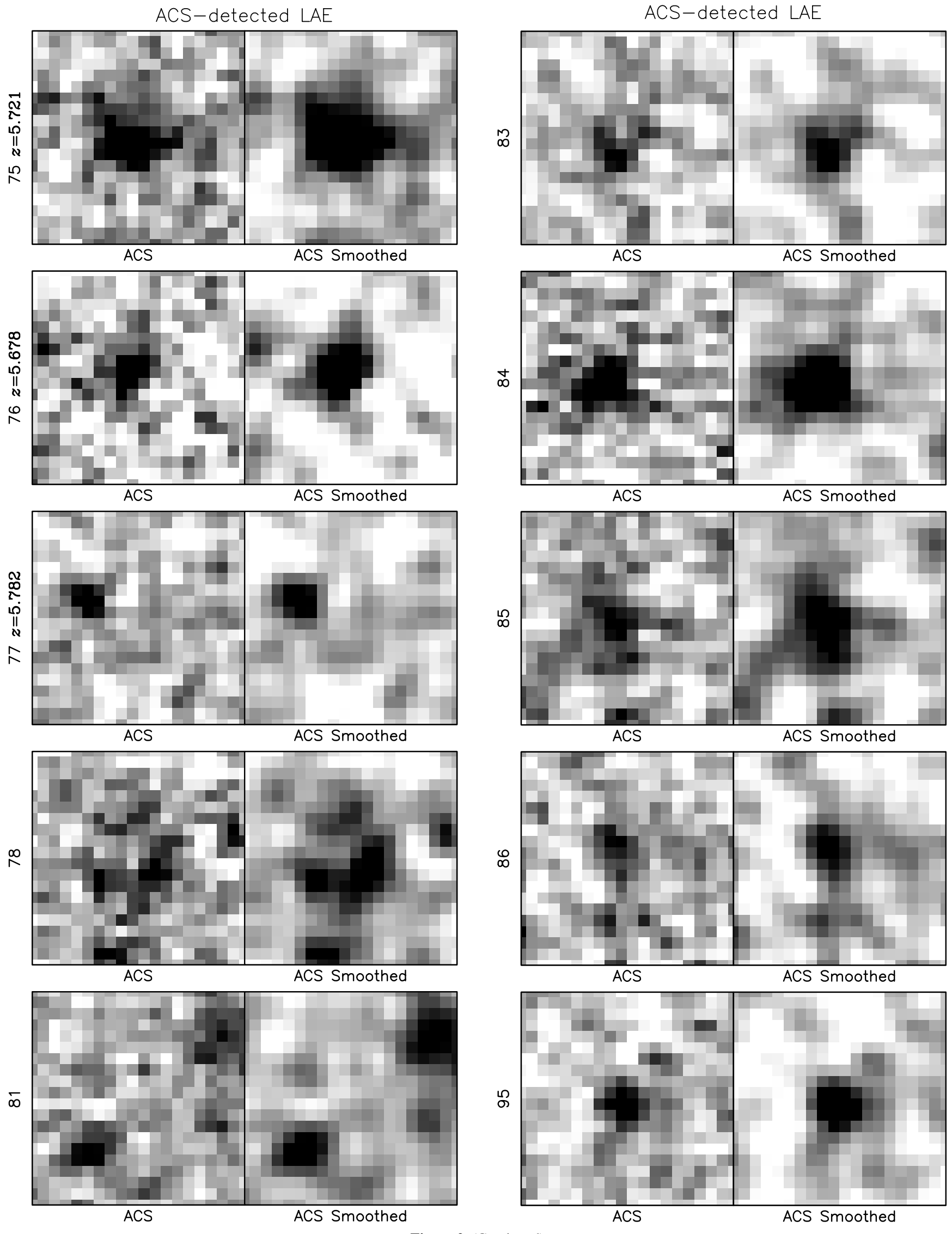

Figure 2. (Continued) 

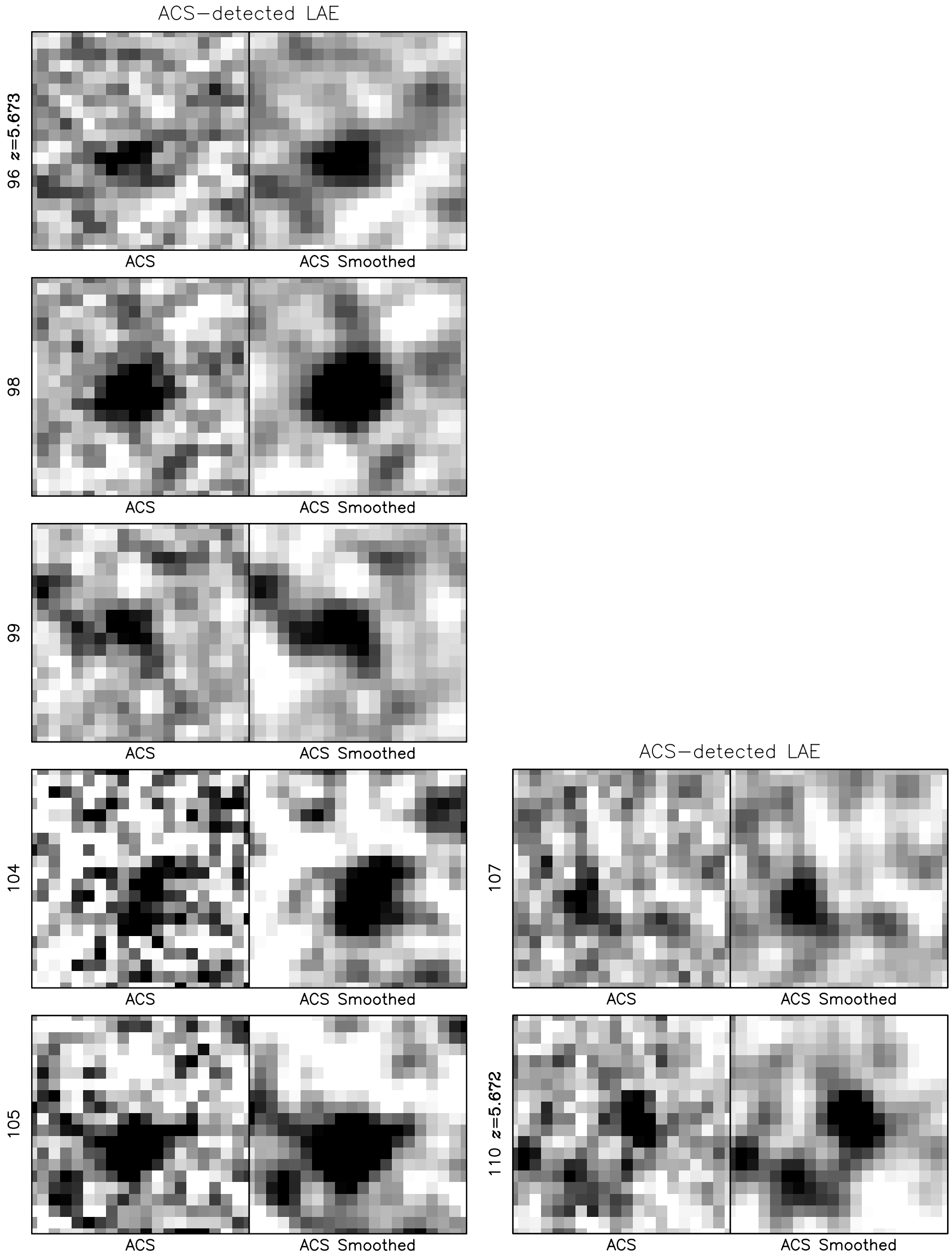

Figure 2. (Continued) 

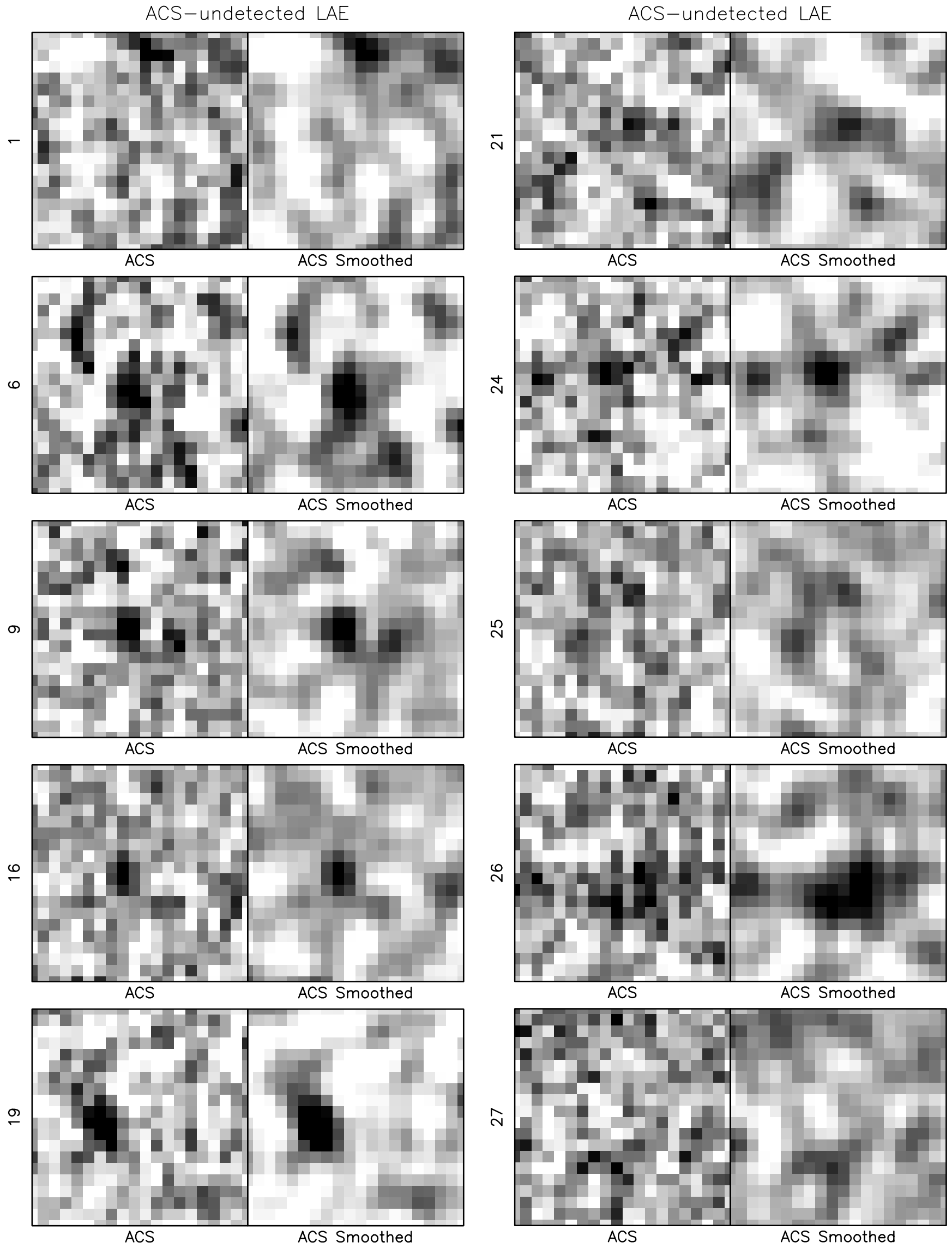

Figure 2. (Continued) 

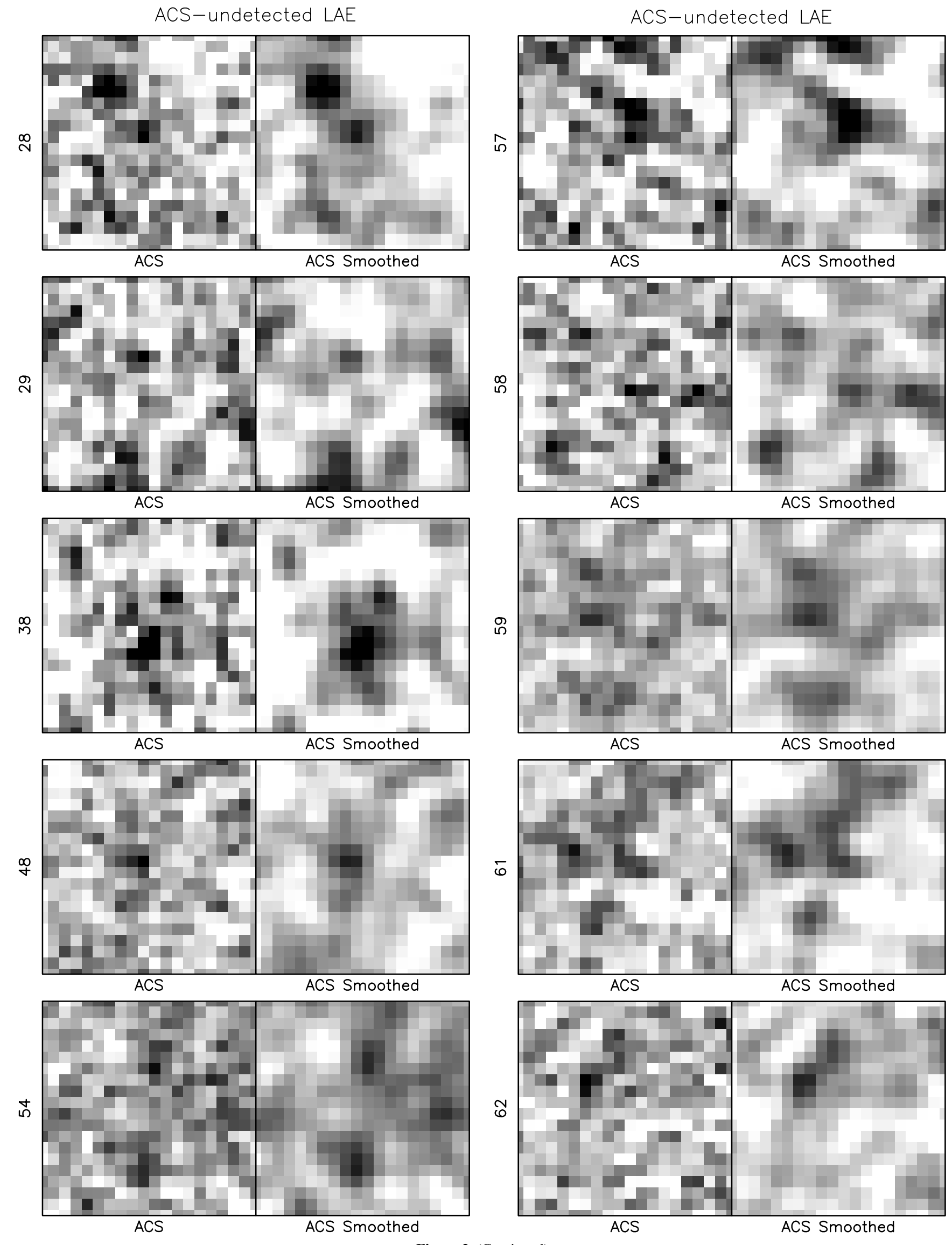

Figure 2. (Continued) 

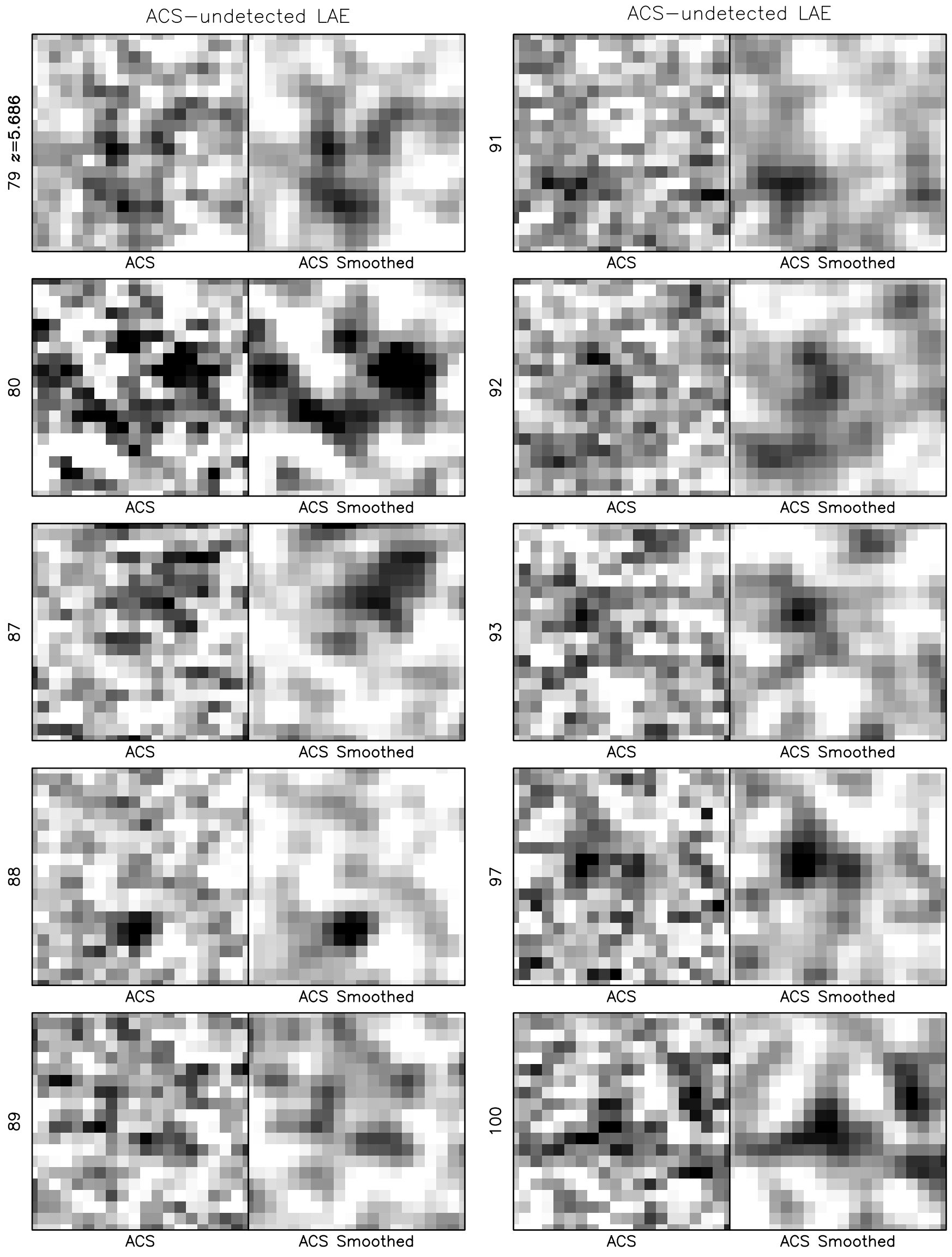

Figure 2. (Continued) 

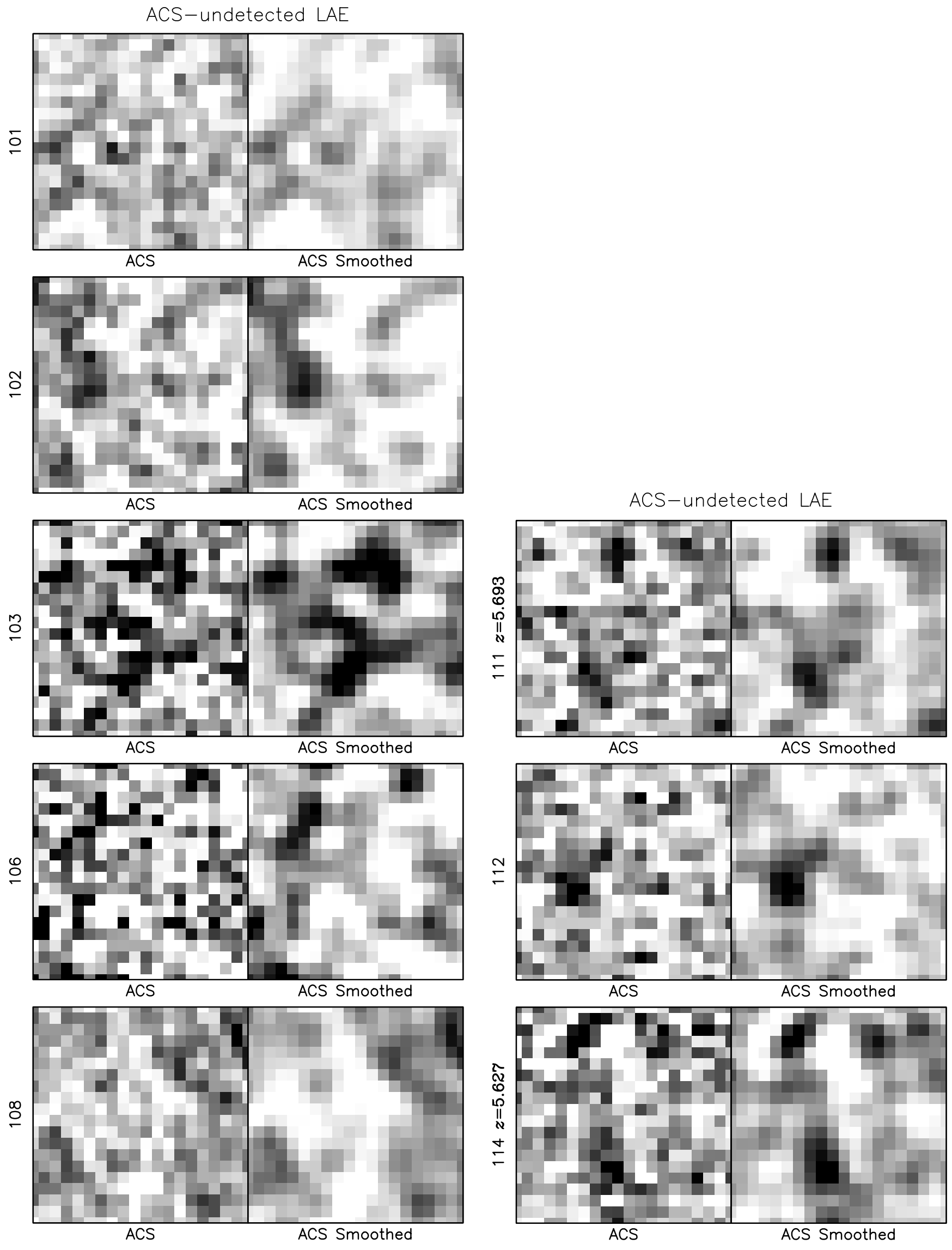

Figure 2. (Continued) 
(a)
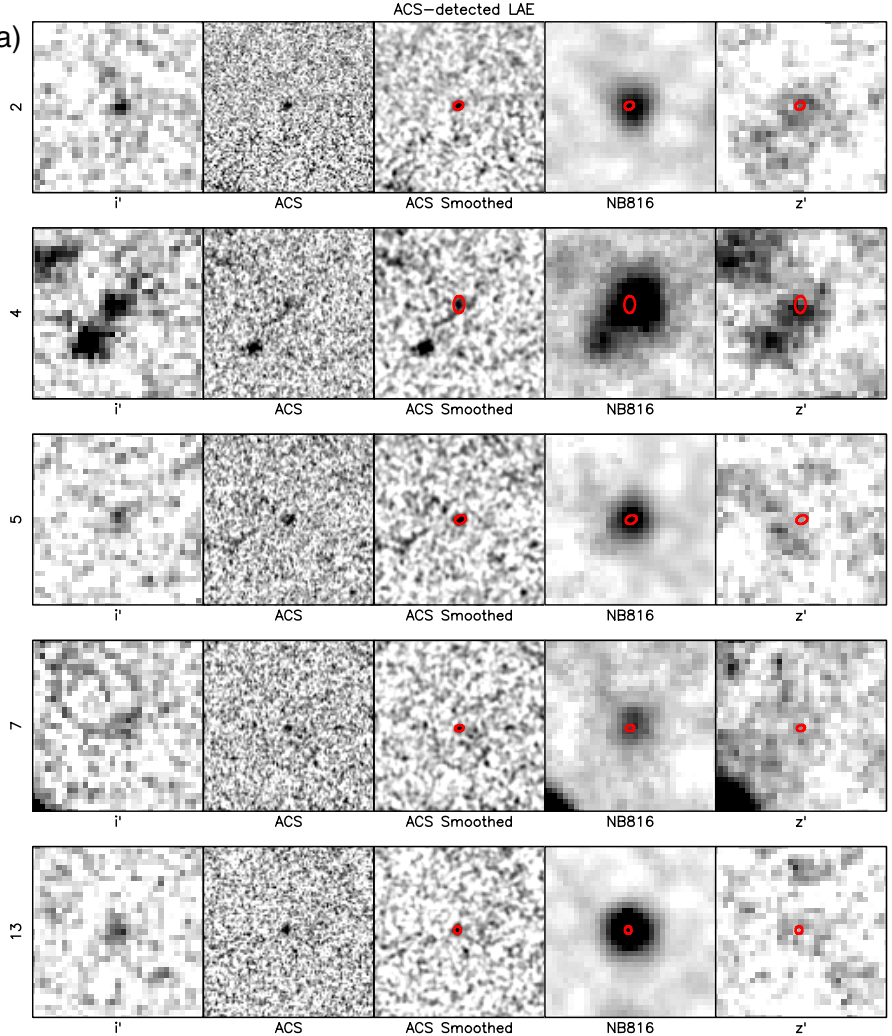

ACS-detected LAE
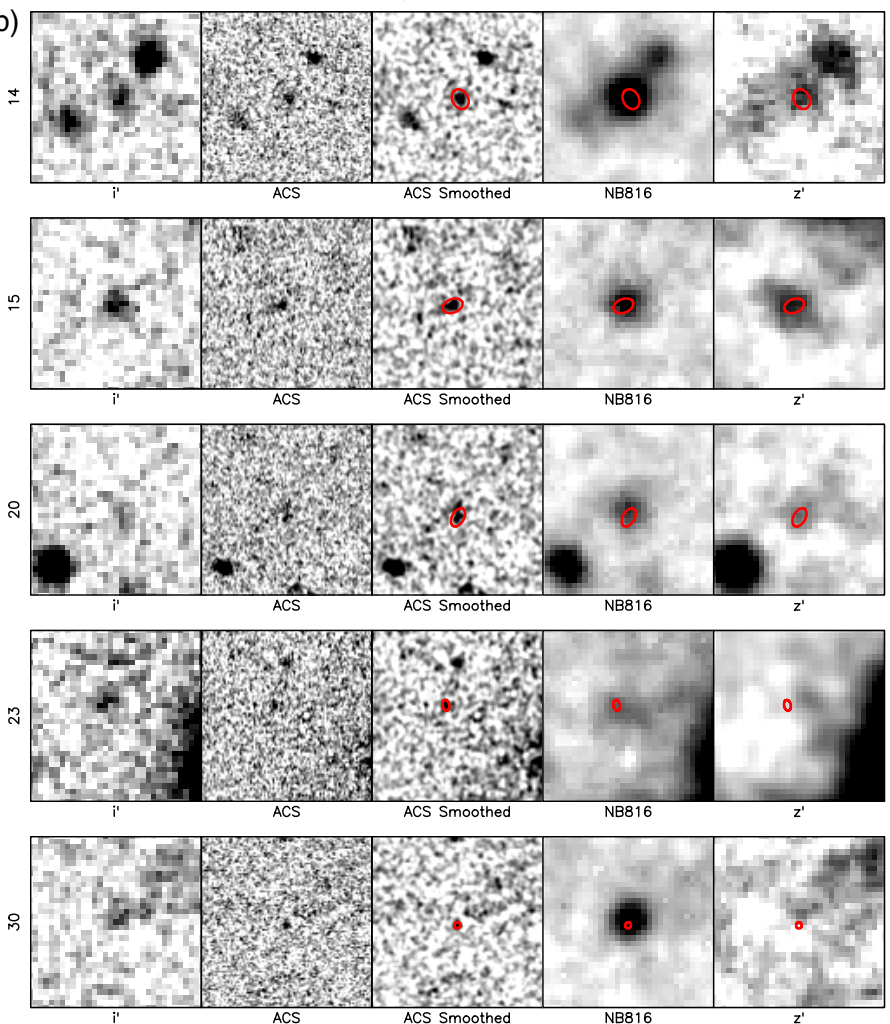

Figure 3. Thumbnails of all 85 LAEs analyzed in this paper. Each panel has a size of $5^{\prime \prime} \times 5^{\prime \prime}$. Red ellipses overplotted on the smoothed ACS, NB816, and $z^{\prime}$ images are half-light ellipses of the detected LAE components in the ACS image. Green and blue ellipses are ACS sources excluded from the sample by eye inspection and by rejection of foreground neighbors, respectively.

the ACS-undetected LAEs, we show $3 \sigma$ upper limits within a $1^{\prime \prime}$ diameter aperture in Figure 6. If we adopt $1 \sigma$ and $2 \sigma$ upper limits, the limiting magnitudes are fainter by 1.2 and $0.45 \mathrm{mag}$, respectively.
In order to examine the effect of limiting surface brightness in our ACS imaging, we show the relation between $R_{\mathrm{HL}}$ and $I_{814}$ magnitude in Figure 7. In this diagram, we find no faint object with a large $R_{\mathrm{HL}}$. This suggests that we are unable to detect 
(c)
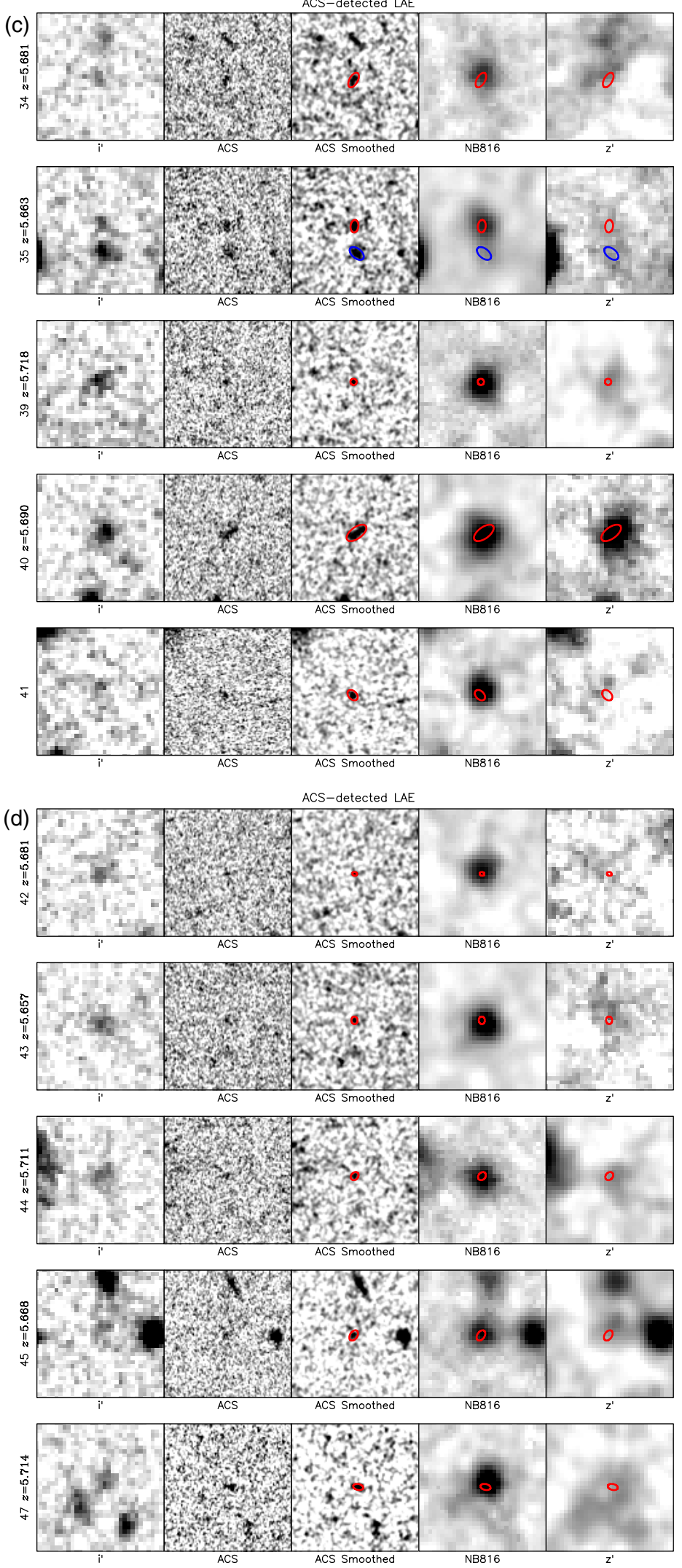

Figure 3. (Continued)

LAEs with $R_{\mathrm{HL}}>0.5$ arcsec if they are fainter than 26 mag in $I_{814}$.

In Figure 8, the frequency distributions of sizes in NB816 imaging, FWHM (NB816), are shown for the ACS-detected and
ACS-undetected LAEs. Not a small part of the sample LAEs have larger sizes than the PSF size $(0.98$ arcsec $)$, especially for the ACS-undetected LAEs. Their appearance in the NB816 images shows widely extended morphology (e.g., No. 74 in the 

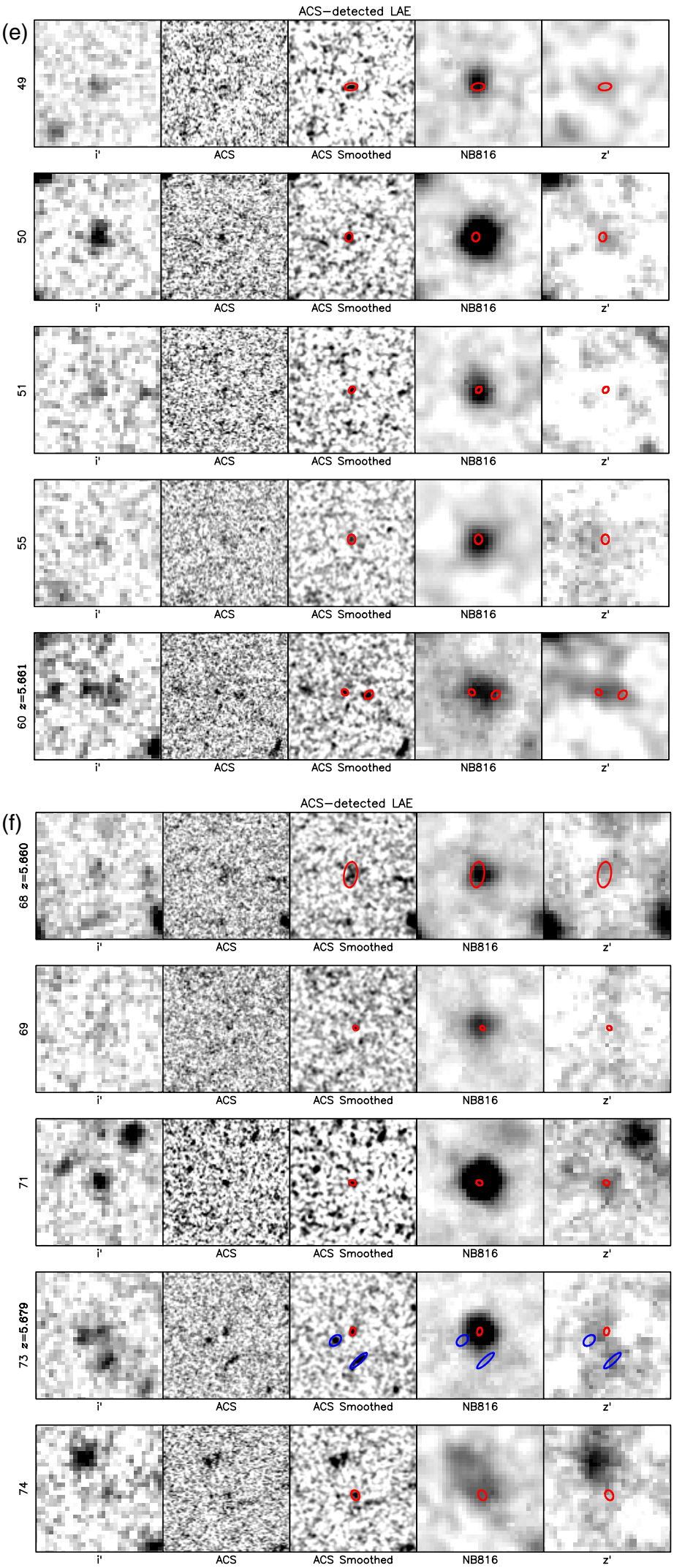

Figure 3. (Continued)

ACS-detected subsample, and No. 1, No. 27, and No. 29 in the ACS-undetected subsample) and looks like a so-called Ly $\alpha$ blob (e.g., Fynbo et al. 1999; Steidel et al. 2000; Matsuda et al. 2004; Saito et al. 2006). In Figure 9, we also show the diagram between
FWHM (NB816) and $R_{\mathrm{HL}}$. This tendency is more conspicuous for the ACS-undetected LAEs. Even if a LAE has a larger size in NB816, i.e., it has an extended Ly $\alpha$ source, we would be able to detect it in our ACS F814W imaging if it has a compact 

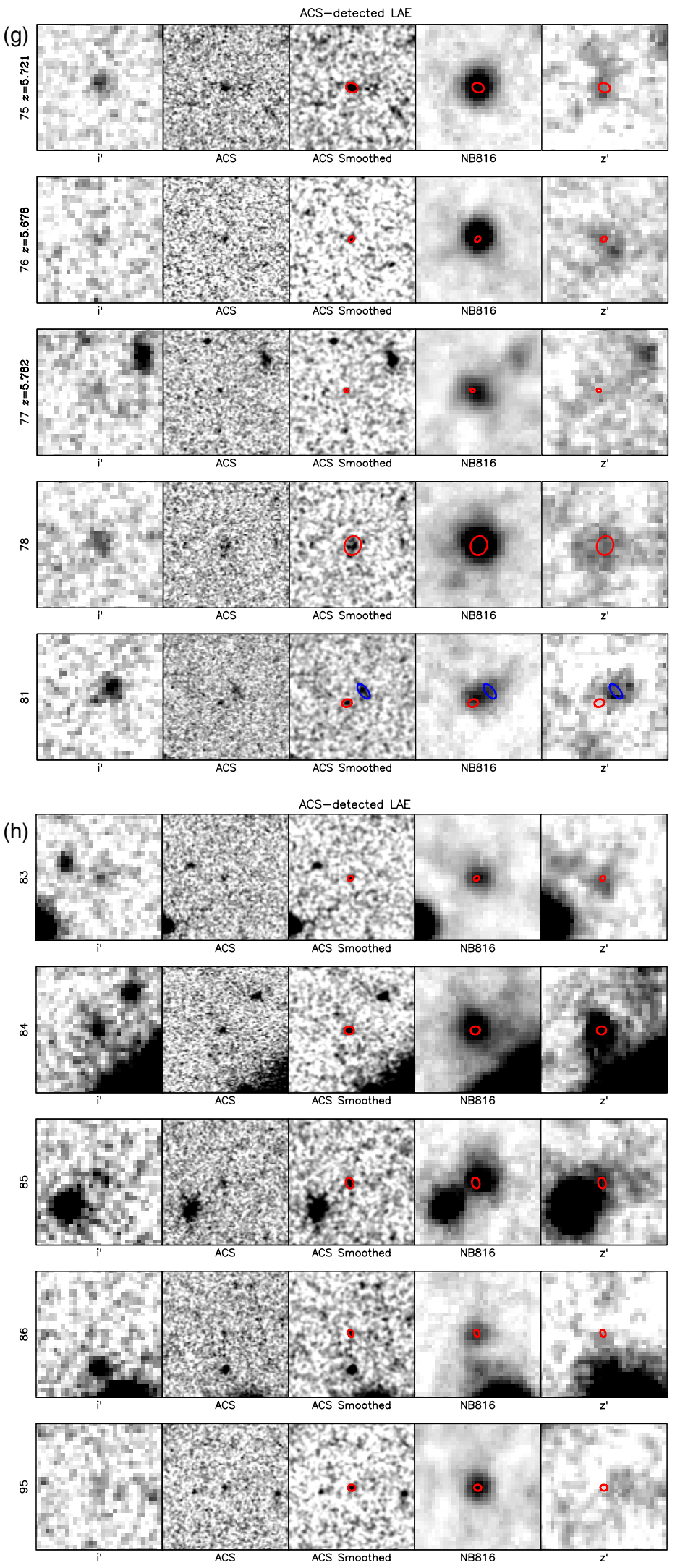

Figure 3. (Continued)

UV continuum component less than 0.5 arcsec. Therefore, it is suggested that the ACS-undetected LAEs have an extended UV continuum component larger than 0.5 arcsec. However, the $z^{\prime}$ magnitudes of the ACS-undetected LAEs are typically fainter relative to the ones detected in ACS (see Table 3). This means that their UV magnitudes are in some cases too faint to be detected in our ACS imaging even though they are not very much extended (i.e., $<0.5$ arcsec); see Section 3.4 for more details. 

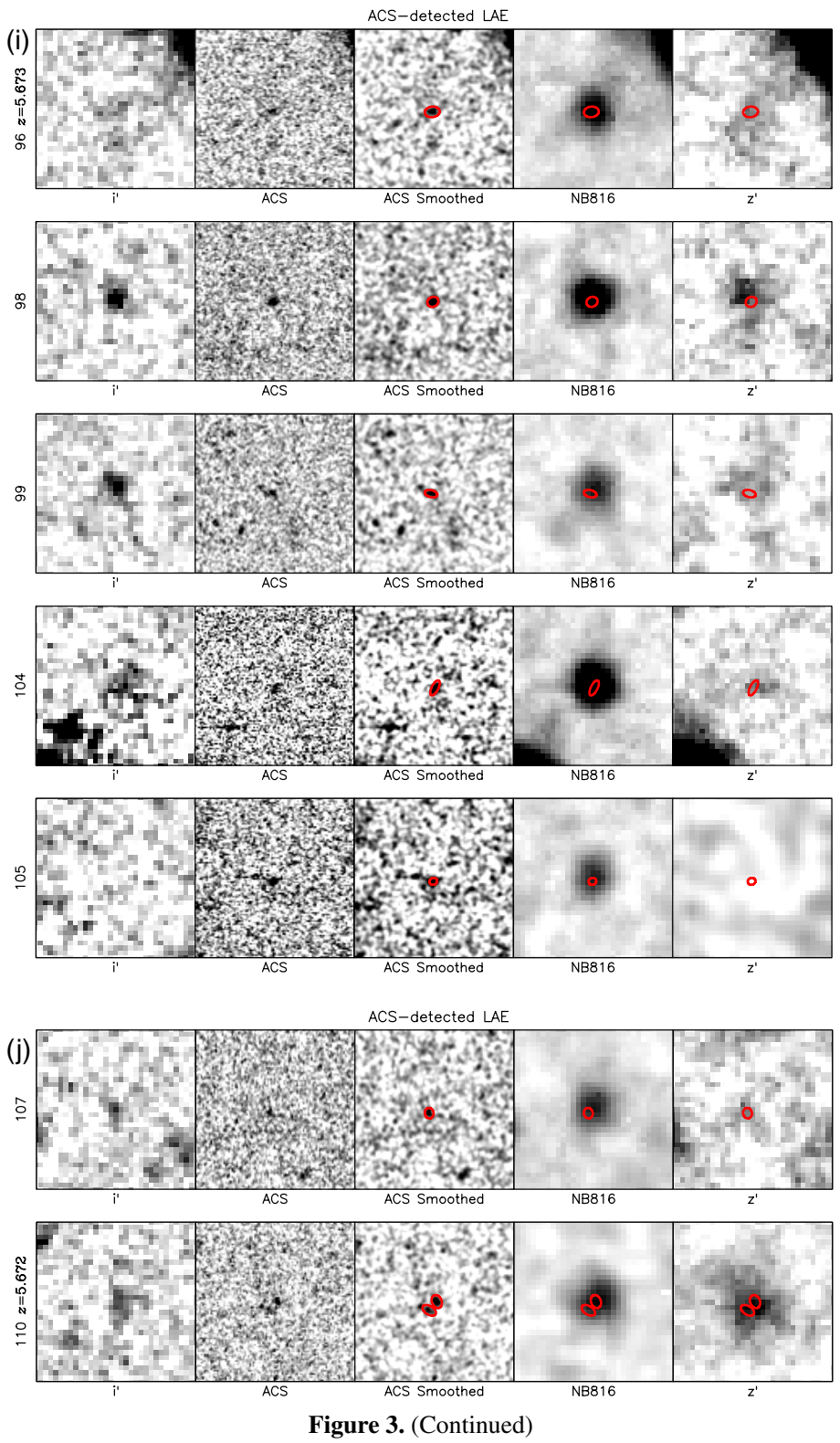

\subsection{Monte Carlo Simulations}

To estimate practical errors in our measurements of $R_{\mathrm{HL}}$, we performed Monte Carlo simulations. We prepared 1000 artificial sources with the exponential light profile for each set of given parameters of the total magnitude, the half-light radius, and the ellipticity. We put these sources on the observed ACS image after they were convolved with the PSF image. We also added photon noises. Then we measured their photometric properties with the same SExtractor parameters for the source detection. Detection completeness of the $50 \%$ limit is indicated in Figure 7.

Based on these simulations, we estimated probability distributions of each parameter for each LAE. The median values with the $68 \%$ confidence intervals of estimated distributions for total magnitude and half-light radius are listed in Table 3. In the upper panel of Figure 10, we show the relation between estimated magnitudes and measured magnitudes. The estimated magnitudes tend to be brighter than the measured magnitudes. The estimated errors are typically \pm 0.3 to \pm 0.5 larger than the typical measured error of \pm 0.1 to \pm 0.2 . Although the estimated half-light radius suffers from large uncertainty, the LAEs mea- sured with $R_{\mathrm{HL}} \geqslant 0.3$ still have a large estimated value in $R_{\mathrm{HL}}$. Moreover, the simulation indicates that several LAEs are intrinsically large even if their measured sizes are nearly the PSF size. While most of LAEs are unresolved objects, we may conclude that some of LAEs at $z \sim 5.7$ have larger intrinsic $R_{\mathrm{HL}}$ than the PSF size.

\subsection{What Do We See in ACS F814W Images: Ly 2 Emission or Ultraviolet Continuum?}

As we see in Section 3.1, the 47 LAEs detected in our ACS F814W imaging are not largely extended (i.e., $R_{\mathrm{HL}}<0.4$ arcsec). What do we see exactly in the ACS images? Here, we examine whether the detected light is from Ly $\alpha$ emission or ultraviolet (UV) continuum.

First, we present the transmission curves for the filters used in our analysis: F814W for ACS/HST and $i^{\prime}$, NB816, and $z^{\prime}$ for Suprime-Cam/Subaru in Figure 11. The CCD sensitivity is taken into account for each filter transmission curve. The bandpass of the NB816 filter is almost centered in the wavelength range covered by the F814W filter. Therefore, 
(k)
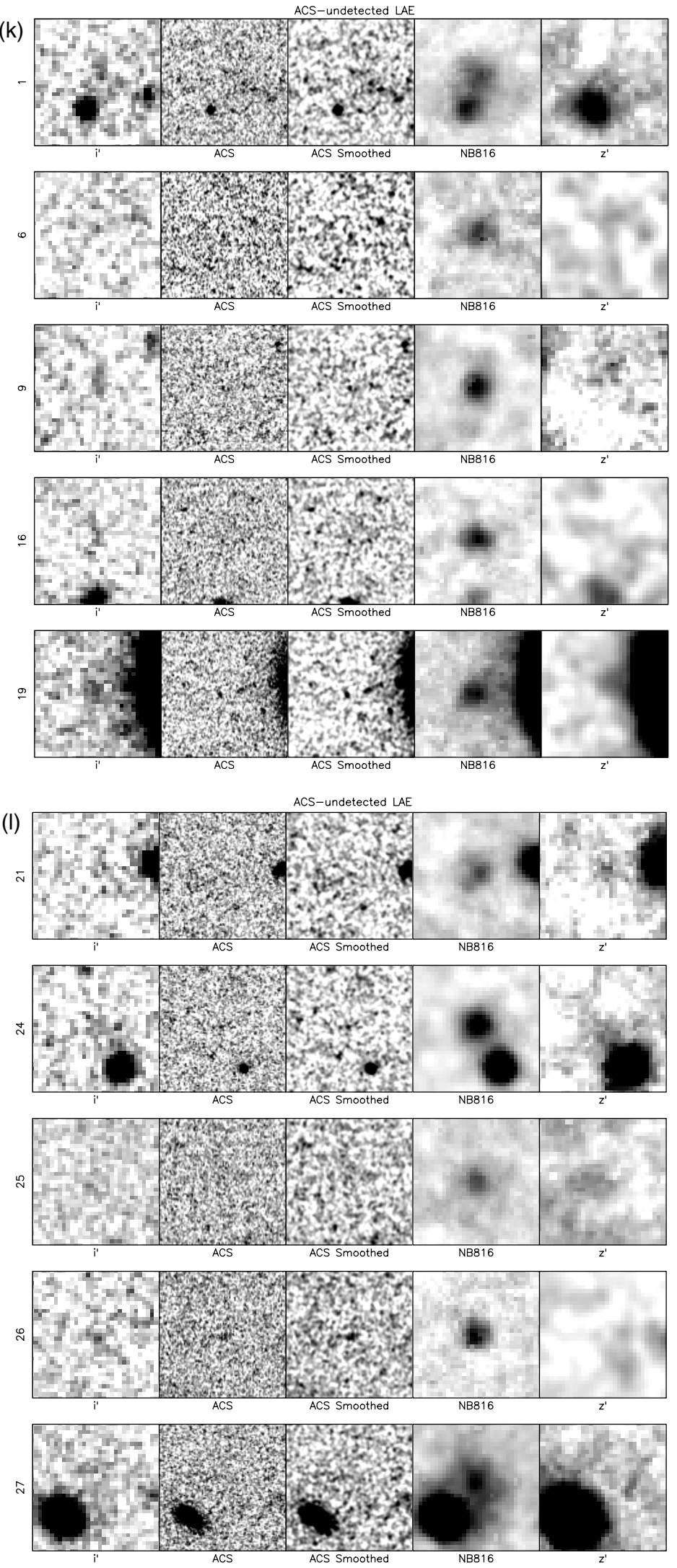

Figure 3. (Continued)

if $\operatorname{Ly} \alpha$ emission is strong enough to be detected in our F814W imaging, we would see Ly $\alpha$ morphologies of LAEs. However, the UV continuum at wavelengths longer than $1216 \AA$ can also be probed by the $\mathrm{F} 814 \mathrm{~W}$ imaging whose transmission curve is similar to the sum of Suprime-Cam $i^{\prime}+z^{\prime}$ filter transmission. In order to investigate what our F814W imaging 

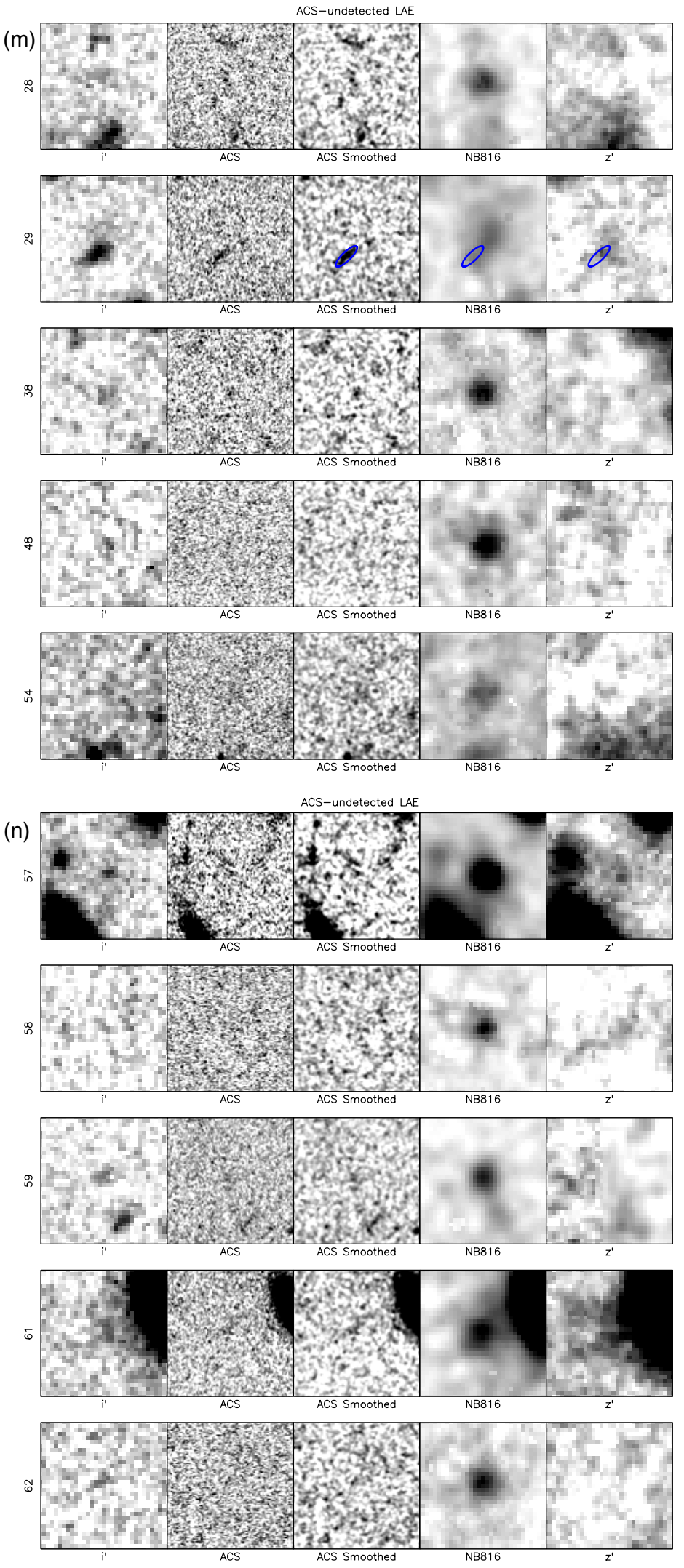

Figure 3. (Continued)

probes, we examine the correlation between $I_{814}$ and NB816 in Figure 12. Since the correlation appears to be poor (its correlation coefficient is $r=0.40$ ), the F814W imaging does not primarily probe Ly $\alpha$ emission. Next, in Figure 13, we show the correlations between $I_{814}$ and $i^{\prime}(r=0.32)$ and $I_{814}$ and $z^{\prime}(r=0.45)$. These comparisons show that $I_{814}$ is more correlated with $z^{\prime}$. This suggests that the F814W imaging probes UV continuum from massive stars in each 

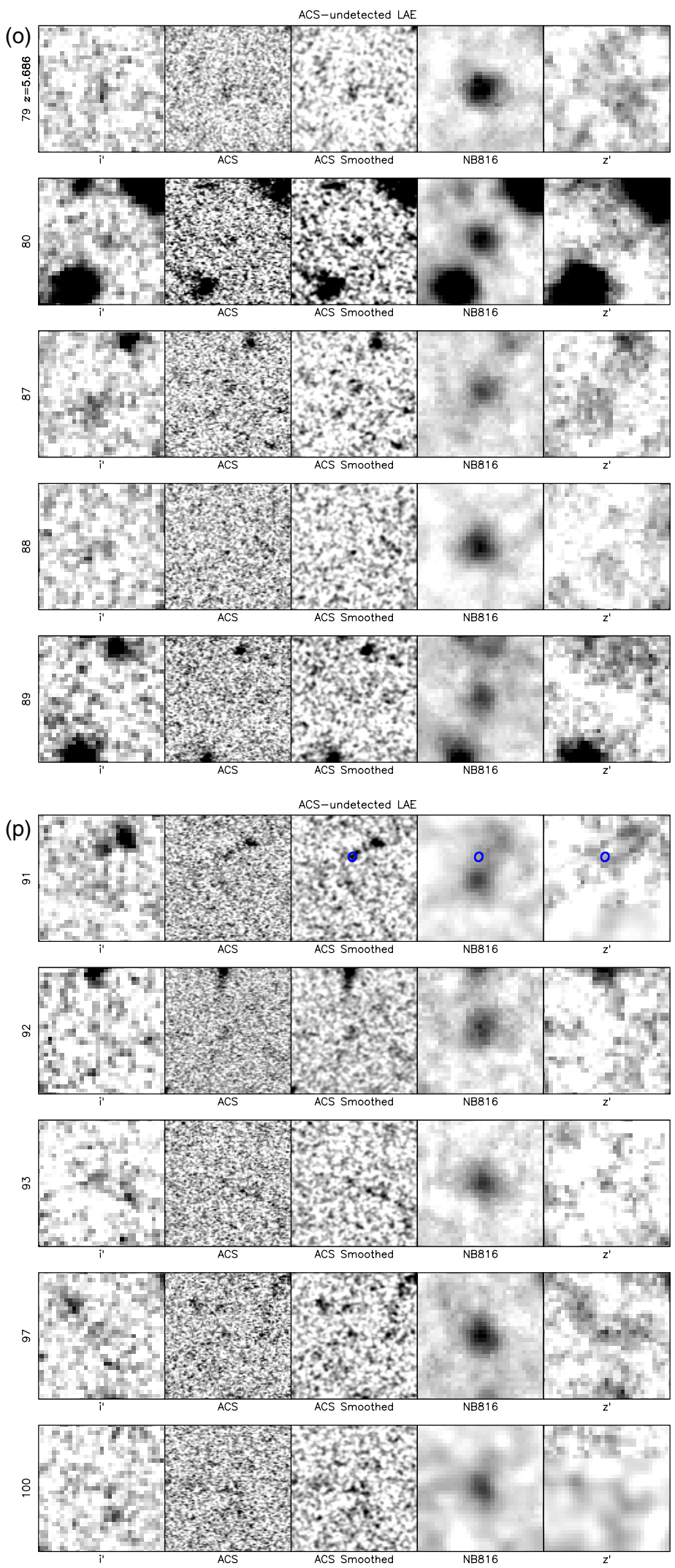

Figure 3. (Continued)

LAE, because $z^{\prime}$ is only sensitive to UV continuum as evident from the filter curves (Figure 11).

Although the correlation between $I_{814}$ and $z^{\prime}$ is better than for NB816 and $i^{\prime}$, there is a systematic offset between $I_{814}$ and $z^{\prime}$ values. $I_{814}$ is typically 0.94 mag (average) fainter than $z^{\prime}$. This offset can be explained by the difference of their filter transmission curves between $I_{814}$ and $z^{\prime}$ (see Figure 11). Since the Ly $\alpha$ wavelength, $1216 \AA$, is observed nearly at the band 

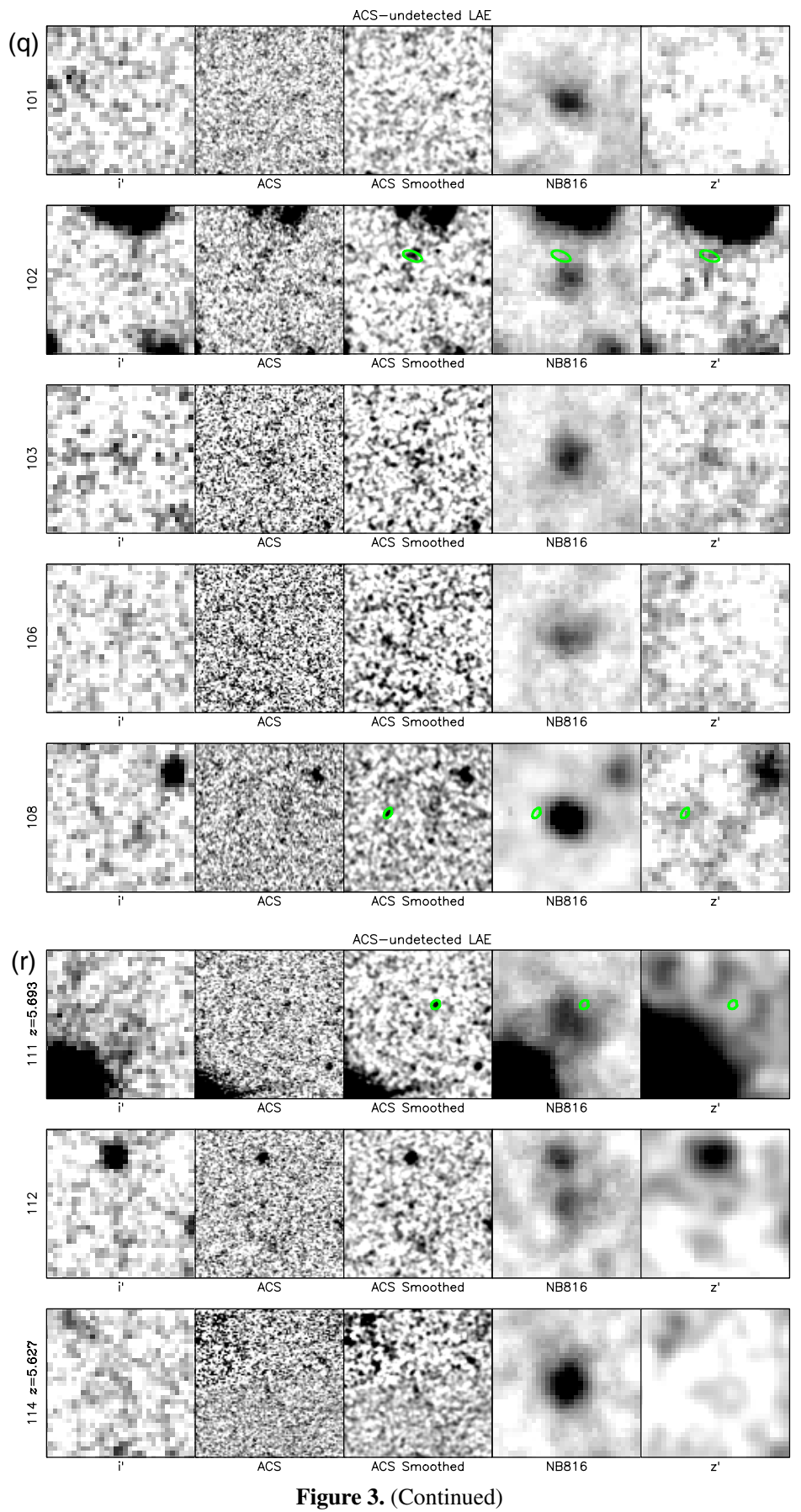

center of the F814W filter, the UV continuum at wavelengths longer than $1216 \AA$ is covered by only half of the F814W bandpass (see Figure 11). As a result, flux densities of the UV continuum are underestimated with our F814W imaging by roughly $0.75(=2.5 \log 2) \mathrm{mag}$. For a more precise treatment, we can estimate the correction factor that converts $I_{814}$ to $z^{\prime}$ for each LAE by assuming a simple model spectrum similar to the one shown in Figure 11, in which $f_{v}$ is assumed to be constant. Given both the UV continuum flux estimated by our $z^{\prime}$ magnitude and the rest-frame EW ( $\left.\operatorname{Ly} \alpha\right)$, we estimate the correction factor for each LAE and then estimate $z^{\prime}$ magnitude based on $I_{814}$ value, $z^{\prime}\left(I_{814}\right)$. For the LAEs with only lower limits of EW $(\operatorname{Ly} \alpha)$, we use the lower limit values for this estimate. In Figure 14, we compare $z^{\prime}\left(I_{814}\right)$ with $z^{\prime}$, and find that there is a tighter correlation $(r=0.58)$ between these two magnitudes. Therefore, we conclude that we see the UV continuum of LAEs and miss almost all the Ly $\alpha$ flux in our ACS imaging.

\subsection{Spatial Sizes of the LAEs Without ACS Counterparts}

Thirty-eight LAEs are not detected in our ACS imaging. This means that the surface brightness of these LAEs is too low to be detected not only in Ly $\alpha$ but also in their UV continuum. Even for LAEs with bright UV continuum, they could not be detected in the ACS image if they are spatially extended and thus their surface brightness falls below the detection limit. Therefore, some of the LAEs that are undetected in the ACS images may have large $R_{\mathrm{HL}}$. 
Table 3

ACS F814W Properties for the LAEs at $z \approx 5.7$

\begin{tabular}{|c|c|c|c|c|}
\hline Number $^{\mathrm{a}}$ & $\begin{array}{l}I_{814^{\mathrm{b}}} \\
(\mathrm{mag})\end{array}$ & $\begin{array}{c}R_{\mathrm{HL}}{ }^{\mathrm{c}} \\
(\operatorname{arcsec})\end{array}$ & $\begin{array}{c}I_{814}{\text { (Estimated })^{\mathrm{d}}}^{(\mathrm{mag})} \\
\end{array}$ & $\begin{array}{c}R_{\mathrm{HL}}(\text { Estimated })^{\mathrm{d}} \\
(\operatorname{arcsec})\end{array}$ \\
\hline \multicolumn{5}{|c|}{ ACS-detected LAEs } \\
\hline 2 & $26.9 \pm 0.1$ & 0.11 & $26.8_{-0.3}^{+0.4}$ & $0.10_{-0.05}^{+0.10}$ \\
\hline 4 & $26.5 \pm 0.1$ & 0.20 & $26.1_{-0.7}^{+0.3}$ & $0.35_{-0.18}^{+0.05}$ \\
\hline 5 & $26.7 \pm 0.1$ & 0.13 & $26.6_{-0.4}^{+0.4}$ & $0.13_{-0.05}^{+0.10}$ \\
\hline 7 & $27.5 \pm 0.2$ & 0.10 & $27.3_{-0.6}^{+0.4}$ & $0.08_{-0.05}^{+0.18}$ \\
\hline 13 & $26.8 \pm 0.1$ & 0.09 & $26.7_{-0.2}^{+0.3}$ & $0.08_{-0.05}^{+0.08}$ \\
\hline 14 & $25.7 \pm 0.1$ & 0.24 & $25.4_{-0.4}^{+0.5}$ & $0.38_{-0.15}^{+0.08}$ \\
\hline 15 & $26.0 \pm 0.1$ & 0.24 & $25.9_{-0.4}^{+0.5}$ & $0.25_{-0.10}^{+0.20}$ \\
\hline 20 & $26.4 \pm 0.1$ & 0.23 & $26.1_{-0.7}^{+0.8}$ & $0.38_{-0.20}^{+0.35}$ \\
\hline 23 & $27.1 \pm 0.2$ & 0.13 & $27.0_{-0.6}^{+0.6}$ & $0.13_{-0.08}^{+0.23}$ \\
\hline 30 & $27.0 \pm 0.1$ & 0.07 & $26.9_{-0.3}^{+0.3}$ & $0.08_{-0.05}^{+0.08}$ \\
\hline 34 & $26.4 \pm 0.1$ & 0.25 & $26.2_{-0.5}^{+0.5}$ & $0.28_{-0.13}^{+0.205}$ \\
\hline 35 & $26.3 \pm 0.10$ & 0.20 & $26.2_{-0.3}^{+0.3}$ & $0.18_{-0.08}^{+0.13}$ \\
\hline 39 & $26.8 \pm 0.1$ & 0.10 & $26.7_{-0.3}^{+0.4}$ & $0.10_{-0.08}^{+0.08}$ \\
\hline 40 & $25.4 \pm 0.1$ & 0.36 & $25.3_{-0.3}^{+0.6}$ & $0.43_{-0.20}^{+0.18}$ \\
\hline 41 & $26.3 \pm 0.1$ & 0.19 & $26.3_{-0.5}^{+0.4}$ & $0.18_{-0.08}^{+0.15}$ \\
\hline 42 & $27.7 \pm 0.1$ & 0.08 & $27.5_{-0.3}^{+0.4}$ & $0.05_{-0.03}^{+0.05}$ \\
\hline 43 & $27.3 \pm 0.2$ & 0.12 & $27.0_{-0.9}^{+0.9}$ & $0.33_{-0.20}^{+0.40}$ \\
\hline 44 & $27.1 \pm 0.2$ & 0.14 & $26.9_{-0.8}^{+0.6}$ & $0.18_{-0.10}^{+0.33}$ \\
\hline 45 & $26.6 \pm 0.1$ & 0.18 & $26.4_{-0.8}^{+0.6}$ & $0.28_{-0.15}^{+0.33}$ \\
\hline 47 & $26.1 \pm 0.1$ & 0.16 & $26.0_{-0.2}^{+0.3}$ & $0.15_{-0.05}^{+0.08}$ \\
\hline 49 & $26.5 \pm 0.2$ & 0.20 & $26.0_{-0.8}^{+0.7}$ & $0.38_{-0.18}^{+0.33}$ \\
\hline 50 & $26.4 \pm 0.1$ & 0.14 & $26.4_{-0.3}^{+0.3}$ & $0.13_{-0.08}^{+0.08}$ \\
\hline 51 & $26.9 \pm 0.2$ & 0.11 & $26.9_{-0.5}^{+0.5}$ & $0.13_{-0.08}^{+0.18}$ \\
\hline 55 & $27.1 \pm 0.2$ & 0.15 & $26.5_{-0.9}^{+1.0}$ & $0.25_{-0.13}^{+0.35}$ \\
\hline $60^{e}$ & $26.8^{f}$ & $0.94^{\mathrm{g}}$ & $\ldots$ & $\ldots$ \\
\hline $60 \mathrm{a}$ & $26.9 \pm 0.2$ & 0.15 & $26.7_{-0.8}^{+0.7}$ & $0.23_{-0.13}^{+0.35}$ \\
\hline $60 \mathrm{~b}$ & $27.3 \pm 0.2$ & 0.11 & $27.1_{-0.6}^{+0.5}$ & $0.10_{-0.05}^{+0.20}$ \\
\hline 68 & $25.9 \pm 0.1$ & 0.40 & $25.7_{-0.6}^{+0.5}$ & $0.35_{-0.13}^{+0.33}$ \\
\hline 69 & $27.8 \pm 0.2$ & 0.08 & $27.5_{-0.4}^{+0.0}$ & $0.03_{-0.03}^{+0.13}$ \\
\hline 71 & $26.1 \pm 0.1$ & 0.10 & $26.1_{-0.1}^{+0 . .^{+}}$ & $0.08_{-0.05}^{+0.05}$ \\
\hline 73 & $26.8 \pm 0.1$ & 0.13 & $26.7_{-0.4}^{+0.4}$ & $0.13_{-0.05}^{+0.13}$ \\
\hline 74 & $26.9 \pm 0.2$ & 0.16 & $26.6_{-1.2}^{+1.2}$ & $0.35_{-0.23}^{+0.40}$ \\
\hline 75 & $26.0 \pm 0.1$ & 0.19 & $26.0_{-0.3}^{+0.3}$ & $0.15_{-0.05}^{+0.10}$ \\
\hline 76 & $26.7 \pm 0.1$ & 0.10 & $26.7_{-0.3}^{+0.3}$ & $0.08_{-0.05}^{+0.08}$ \\
\hline 77 & $27.7 \pm 0.1$ & 0.07 & $27.5_{-0.3}^{+0.4}$ & $0.05_{-0.03}^{+0.05}$ \\
\hline 78 & $25.7 \pm 0.1$ & 0.30 & $25.4_{-0.4}^{+0.5}$ & $0.35_{-0.13}^{+0.25}$ \\
\hline 81 & $27.1 \pm 0.1$ & 0.16 & $26.9_{-0.9}^{+0.4}$ & $0.28_{-0.18}^{+0.43}$ \\
\hline 83 & $27.3 \pm 0.1$ & 0.09 & $27.2_{-0.4}^{+0.4}$ & $0.08_{-0.05}^{+0.13}$ \\
\hline 84 & $26.2 \pm 0.1$ & 0.15 & $26.2_{-0.3}^{+0.3}$ & $0.13_{-0.05}^{+0.08}$ \\
\hline 85 & $26.5 \pm 0.1$ & 0.17 & $26.4_{-0.3}^{+0.3}$ & $0.15_{-0.05}^{+0.10}$ \\
\hline 86 & $27.6 \pm 0.2$ & 0.12 & $27.3_{-0.9}^{+0.3}$ & $0.10_{-0.05}^{+0.05}$ \\
\hline 95 & $27.0 \pm 0.1$ & 0.11 & $26.9_{-0.4}^{+0.4}$ & $0.10_{-0.05}^{+0.10}$ \\
\hline 96 & $26.6 \pm 0.1$ & 0.19 & $26.4_{-0.7}^{+0.6}$ & $0.25_{-0.13}^{+0.30}$ \\
\hline 98 & $26.0 \pm 0.1$ & 0.14 & $26.0_{-0.2}^{+0.3}$ & $0.10_{-0.05}^{+0.08}$ \\
\hline 99 & $26.8 \pm 0.1$ & 0.16 & $26.6_{-0.4}^{+0.5}$ & $0.18_{-0.08}^{+0.10}$ \\
\hline 104 & $26.5 \pm 0.2$ & 0.20 & $26.3_{-0.7}^{+0.7}$ & $0.30_{-0.15}^{+0.35}$ \\
\hline 105 & $25.6 \pm 0.1$ & 0.10 & $25.6_{-0.1}^{+0.1}$ & $0.08_{-0.05}^{+0.03}$ \\
\hline 107 & $27.0 \pm 0.2$ & 0.13 & $26.8_{-0.9}^{+0.7}$ & $0.20_{-0.13}^{+0.35}$ \\
\hline $110^{\mathrm{e}}$ & $26.4^{\mathrm{f}}$ & $0.36^{\mathrm{g}}$ & $\ldots$ & $\ldots$ \\
\hline $110 \mathrm{a}$ & $26.5 \pm 0.1$ & 0.17 & $26.5_{-0.5}^{+0.4}$ & $0.18_{-0.10}^{+0.13}$ \\
\hline $110 \mathrm{~b}$ & $27.1 \pm 0.2$ & 0.18 & $26.8_{-0.9}^{+0.7}$ & $0.28_{-0.15}^{+0.35}$ \\
\hline \multicolumn{5}{|c|}{ ACS-undetected LAEs } \\
\hline 1 & $>27.4$ & $\ldots$ & $\ldots$ & $\cdots$ \\
\hline 6 & $>27.1$ & $\ldots$ & $\ldots$ & $\ldots$ \\
\hline 9 & $>27.3$ & $\ldots$ & $\cdots$ & $\cdots$ \\
\hline 16 & $>27.4$ & $\cdots$ & $\cdots$ & $\cdots$ \\
\hline 19 & $>27.2$ & $\ldots$ & $\ldots$ & $\ldots$ \\
\hline 21 & $>27.4$ & $\cdots$ & $\cdots$ & $\cdots$ \\
\hline 24 & $>27.4$ & $\ldots$ & $\ldots$ & $\ldots$ \\
\hline 25 & $>27.5$ & $\cdots$ & $\cdots$ & $\cdots$ \\
\hline 26 & $>27.4$ & $\ldots$ & $\cdots$ & $\cdots$ \\
\hline
\end{tabular}

Table 3

(Continued)

\begin{tabular}{|c|c|c|c|c|}
\hline Number $^{\mathrm{a}}$ & $\begin{array}{l}I_{814^{b}} \\
(\mathrm{mag})\end{array}$ & $\begin{array}{c}R_{\mathrm{HL}}^{\mathrm{c}} \\
(\operatorname{arcsec})\end{array}$ & $\begin{array}{c}I_{814}(\text { Estimated })^{\mathrm{d}} \\
(\mathrm{mag})\end{array}$ & $\begin{array}{c}R_{\mathrm{HL}}(\text { Estimated })^{\mathrm{d}} \\
(\operatorname{arcsec})\end{array}$ \\
\hline 27 & $>27.3$ & $\ldots$ & $\ldots$ & $\ldots$ \\
\hline 28 & $>27.3$ & $\ldots$ & $\cdots$ & $\ldots$ \\
\hline 29 & $>27.4$ & $\ldots$ & $\ldots$ & $\ldots$ \\
\hline 38 & $>27.3$ & $\ldots$ & $\ldots$ & $\ldots$ \\
\hline 48 & $>27.5$ & $\ldots$ & $\ldots$ & $\ldots$ \\
\hline 54 & $>27.5$ & $\ldots$ & $\cdots$ & $\ldots$ \\
\hline 57 & $>27.1$ & $\cdots$ & $\ldots$ & $\cdots$ \\
\hline 58 & $>27.4$ & $\ldots$ & $\ldots$ & $\ldots$ \\
\hline 59 & $>27.8$ & $\ldots$ & $\ldots$ & $\ldots$ \\
\hline 61 & $>27.5$ & $\cdots$ & $\ldots$ & $\cdots$ \\
\hline 62 & $>27.4$ & $\ldots$ & $\ldots$ & $\ldots$ \\
\hline 79 & $>27.6$ & $\ldots$ & $\ldots$ & $\ldots$ \\
\hline 80 & $>26.9$ & $\ldots$ & $\ldots$ & $\ldots$ \\
\hline 87 & $>27.2$ & $\ldots$ & $\cdots$ & $\ldots$ \\
\hline 88 & $>27.5$ & $\ldots$ & $\cdots$ & $\ldots$ \\
\hline 89 & $>27.3$ & $\ldots$ & $\ldots$ & $\ldots$ \\
\hline 91 & $>27.4$ & $\ldots$ & $\ldots$ & $\ldots$ \\
\hline 92 & $>27.6$ & $\ldots$ & $\cdots$ & $\cdots$ \\
\hline 93 & $>27.3$ & $\cdots$ & $\ldots$ & $\cdots$ \\
\hline 97 & $>27.2$ & $\ldots$ & $\cdots$ & $\ldots$ \\
\hline 100 & $>27.3$ & $\ldots$ & $\cdots$ & $\ldots$ \\
\hline 101 & $>27.6$ & $\ldots$ & $\ldots$ & $\ldots$ \\
\hline 102 & $>27.3$ & $\ldots$ & $\ldots$ & $\ldots$ \\
\hline 103 & $>26.9$ & $\ldots$ & $\cdots$ & $\cdots$ \\
\hline 106 & $>26.7$ & $\cdots$ & $\ldots$ & $\cdots$ \\
\hline 108 & $>27.4$ & $\cdots$ & $\cdots$ & $\cdots$ \\
\hline 111 & $>27.2$ & $\ldots$ & $\cdots$ & $\ldots$ \\
\hline 112 & $>27.3$ & $\ldots$ & $\ldots$ & $\ldots$ \\
\hline 114 & $>27.1$ & $\ldots$ & $\ldots$ & $\ldots$ \\
\hline
\end{tabular}

Notes.

a The LAE IDs given in Murayama et al. (2007).

b AB magnitude. Lower limits represent $3 \sigma$ significance with assuming a $1^{\prime \prime}$ diameter aperture.

${ }^{\mathrm{c}}$ Effective radius.

${ }^{\mathrm{d}}$ Estimated values by Monte Carlo calculations.

e Double ACS sources.

${ }^{f}$ Magnitude corresponding to the sum of flux of each source.

$g$ Separation between the double sources.

Here, assuming that the LAEs without ACS counterparts also have the same SED as that adopted in the previous subsection, we estimate the expected $I_{814}$ magnitude, $I_{814}\left(z^{\prime}\right)$, based on the Subaru $z^{\prime}$ and the NB816 magnitudes. We find that 16 of the 38 ACS-undetected LAEs would be bright as much as $I_{814}\left(z^{\prime}\right) \leqslant 26.5$. For LAEs with $I_{814}=26.5$ and $R_{\mathrm{HL}}=0.15$ arcsec, the detection completeness is $50 \%$ (see Figure 7). Therefore, these 16 LAEs with $I_{814}\left(z^{\prime}\right) \leqslant 26.5$ are probably also slightly extended LAEs $\left(R_{\mathrm{HL}} \geqslant 0.15\right.$ arcsec $)$. However, there is no LAE without ACS counterparts brighter than $25 \mathrm{mag}$ in $I_{814}\left(z^{\prime}\right)$. This suggests that very extended LAEs with $R_{\mathrm{HL}} \geqslant 0.4$ arcsec are not present in our LAE sample.

As shown in the previous subsection, $z^{\prime}$ is well correlated with $I_{814}$ and these magnitudes represent UV continuum brightness for the ACS-detected LAEs. For the ACS-undetected LAEs, it is not uncertain if this relation would be still valid. Their $z^{\prime}$ are systematically fainter than those of the ACS-detected LAEs, while the magnitude ranges of NB816 for both the subsamples are nearly the same, implying that contribution of Ly $\alpha$ flux would be considerable for that of the ACS-undetected LAEs. 
Table 4

Photometric Properties from Subaru Suprime-Cam Images for the LAEs at $z \approx 5.7$

\begin{tabular}{|c|c|c|c|c|c|c|c|c|c|}
\hline Number ${ }^{\mathrm{a}}$ & $\begin{array}{c}i^{\prime \mathrm{b}} \\
\left(\phi 3^{\prime \prime} 0\right)\end{array}$ & $\begin{array}{c}z^{\prime \mathrm{b}} \\
\left(\phi 3^{\prime \prime} 0\right)\end{array}$ & $\begin{array}{c}\mathrm{NB} 816^{\mathrm{b}} \\
\left(\phi 3^{\prime \prime} 0\right)\end{array}$ & $\begin{array}{c}\text { FWHM }(\mathrm{NB} 816)^{\mathrm{c}} \\
(\operatorname{arcsec})\end{array}$ & $\epsilon(\mathrm{NB} 816)^{\mathrm{d}}$ & $\begin{array}{c}\text { PA (NB816) } \\
\text { (degree) }\end{array}$ & $\begin{array}{c}L(\operatorname{Ly} \alpha) \\
\left(10^{42} \mathrm{erg} \mathrm{s}^{-1}\right)\end{array}$ & $\begin{array}{c}E W_{0}(\mathrm{Ly} \alpha)^{\mathrm{e}} \\
(\AA)\end{array}$ & $z_{\mathrm{sp}}{ }^{\mathrm{f}}$ \\
\hline \multicolumn{10}{|c|}{ ACS-detected LAEs } \\
\hline 2 & 26.5 & 25.9 & 24.6 & 1.1 & 0.17 & 9 & 8.0 & 46 & $\cdots$ \\
\hline 4 & 24.5 & 24.8 & 23.2 & 1.4 & 0.08 & 168 & 31.0 & 67 & $\cdots$ \\
\hline 5 & 27.1 & 26.2 & 24.4 & 1.2 & 0.04 & 141 & 10.7 & 86 & $\cdots$ \\
\hline 7 & 26.0 & 25.6 & 24.6 & 1.3 & 0.11 & 179 & 7.9 & 34 & $\cdots$ \\
\hline 13 & 27.6 & 99.0 & 23.9 & 1.1 & 0.07 & 68 & 18.4 & $>175$ & $\cdots$ \\
\hline 14 & 24.9 & 25.0 & 23.6 & 1.4 & 0.15 & 74 & 21.2 & 55 & $\cdots$ \\
\hline 15 & 26.2 & 25.1 & 24.6 & 1.0 & 0.06 & 92 & 6.7 & 19 & $\cdots$ \\
\hline 20 & 26.5 & 26.0 & 24.7 & 1.0 & 0.05 & 106 & 8.0 & 51 & $\cdots$ \\
\hline 23 & 25.4 & 25.9 & 24.4 & 1.4 & 0.37 & 91 & 10.1 & 58 & $\cdots$ \\
\hline 30 & 25.9 & 25.8 & 24.3 & 1.0 & 0.11 & 127 & 12.2 & 66 & $\cdots$ \\
\hline 34 & 26.4 & 25.5 & 24.7 & 1.7 & 0.31 & 8 & 7.3 & 29 & 5.681 \\
\hline 35 & 25.5 & 25.6 & 24.4 & 1.5 & 0.41 & 14 & 9.6 & 42 & 5.663 \\
\hline 39 & 25.9 & 26.0 & 24.2 & 1.1 & 0.16 & 154 & 13.6 & 89 & 5.718 \\
\hline 40 & 25.5 & 24.4 & 23.9 & 1.5 & 0.09 & 174 & 13.3 & 20 & 5.690 \\
\hline 41 & 27.1 & 99.0 & 24.5 & 1.0 & 0.16 & 6 & 11.2 & $>103$ & $\cdots$ \\
\hline 42 & 27.0 & 27.3 & 24.8 & 1.1 & 0.06 & 137 & 8.0 & $>72$ & 5.681 \\
\hline 43 & 26.3 & 26.1 & 24.2 & 1.3 & 0.10 & 166 & 13.2 & 91 & 5.657 \\
\hline 44 & 26.3 & 26.0 & 24.3 & 1.4 & 0.31 & 67 & 11.6 & 77 & 5.711 \\
\hline 45 & 25.7 & 25.9 & 24.5 & 1.4 & 0.20 & 97 & 9.6 & 55 & 5.668 \\
\hline 47 & 25.5 & 25.2 & 24.1 & 1.4 & 0.18 & 128 & 13.5 & 42 & 5.714 \\
\hline 49 & 26.8 & 28.3 & 24.8 & 1.1 & 0.24 & 176 & 8.0 & $>84$ & $\cdots$ \\
\hline 50 & 26.0 & 99.0 & 23.5 & 1.2 & 0.06 & 160 & 27.9 & $>260$ & $\cdots$ \\
\hline 51 & 27.0 & 99.0 & 24.9 & 1.2 & 0.26 & 22 & 9.5 & $>88$ & $\cdots$ \\
\hline 55 & 27.3 & 26.6 & 24.5 & 1.3 & 0.19 & 139 & 9.8 & $>97$ & $\ldots$ \\
\hline 60 & 25.4 & 25.5 & 24.1 & 1.5 & 0.22 & 79 & 13.7 & 56 & 5.661 \\
\hline 68 & 26.4 & 26.7 & 24.5 & 1.0 & 0.06 & 75 & 10.4 & $>111$ & 5.660 \\
\hline 69 & 27.8 & 99.0 & 24.6 & 1.3 & 0.10 & 49 & 10.0 & $>101$ & $\ldots$ \\
\hline 71 & 25.4 & 25.3 & 23.3 & 1.1 & 0.05 & 50 & 31.1 & 105 & $\cdots$ \\
\hline 73 & 25.2 & 25.7 & 24.0 & 1.1 & 0.09 & 14 & 15.0 & 73 & 5.679 \\
\hline 74 & 26.2 & 25.6 & 24.4 & 1.9 & 0.31 & 53 & 9.9 & 44 & $\ldots$ \\
\hline 75 & 26.7 & 26.0 & 24.2 & 1.2 & 0.07 & 147 & 13.4 & 90 & 5.721 \\
\hline 76 & 29.7 & 25.6 & 24.0 & 1.1 & 0.06 & 14 & 14.8 & 64 & 5.678 \\
\hline 77 & 26.2 & 25.6 & 24.3 & 1.2 & 0.10 & 62 & 11.5 & 53 & 5.782 \\
\hline 78 & 25.7 & 25.0 & 23.5 & 1.4 & 0.01 & 164 & 23.8 & 64 & $\cdots$ \\
\hline 81 & 25.7 & 26.4 & 24.6 & 1.5 & 0.36 & 116 & 8.9 & $>76$ & $\cdots$ \\
\hline 83 & 26.0 & 25.6 & 24.7 & 1.2 & 0.19 & 58 & 7.1 & 31 & $\cdots$ \\
\hline 84 & 24.6 & 24.0 & 23.5 & 1.5 & 0.22 & 34 & 18.1 & 19 & $\cdots$ \\
\hline 85 & 25.3 & 24.5 & 23.8 & 1.5 & 0.17 & 19 & 16.4 & 27 & $\cdots$ \\
\hline 86 & 26.7 & 26.3 & 24.8 & 1.2 & 0.20 & 154 & 7.7 & 64 & $\cdots$ \\
\hline 95 & 26.7 & 26.9 & 24.7 & 1.1 & 0.22 & 14 & 8.5 & $>79$ & $\cdots$ \\
\hline 96 & 25.9 & 25.5 & 24.1 & 1.1 & 0.16 & 157 & 13.5 & 56 & 5.673 \\
\hline 98 & 26.1 & 25.5 & 24.0 & 1.1 & 0.06 & 78 & 14.8 & 58 & $\ldots$ \\
\hline 99 & 25.8 & 26.1 & 24.6 & 1.3 & 0.20 & 166 & 8.7 & 64 & $\ldots$ \\
\hline 104 & 25.8 & 26.2 & 23.7 & 1.0 & 0.07 & 22 & 22.3 & 170 & $\cdots$ \\
\hline 105 & 99.0 & 99.0 & 25.1 & 1.1 & 0.24 & 171 & 6.5 & $>59$ & $\cdots$ \\
\hline 107 & 26.5 & 27.7 & 24.6 & 1.3 & 0.20 & 162 & 10.1 & $>92$ & $\cdots$ \\
\hline 110 & 26.2 & 24.7 & 24.3 & 1.2 & 0.05 & 48 & 8.4 & 16 & 5.672 \\
\hline \multicolumn{10}{|c|}{ ACS-undetected LAEs } \\
\hline 1 & 25.8 & 25.9 & 24.5 & 1.7 & 0.31 & 71 & 9.2 & 53 & $\ldots$ \\
\hline 6 & 27.1 & 26.4 & 24.7 & 1.2 & 0.16 & 97 & 8.1 & $>74$ & $\cdots$ \\
\hline 9 & 27.3 & 30.4 & 24.6 & 1.2 & 0.31 & 168 & 10.4 & $>98$ & $\cdots$ \\
\hline 16 & 26.9 & 27.5 & 24.9 & 1.1 & 0.21 & 84 & 7.1 & $>68$ & $\ldots$ \\
\hline 19 & 25.2 & 25.9 & 24.2 & 1.4 & 0.31 & 136 & 13.0 & 77 & $\ldots$ \\
\hline 21 & 26.7 & 27.5 & 24.8 & 1.2 & 0.25 & 136 & 8.4 & $>79$ & $\ldots$ \\
\hline 24 & 26.4 & 26.4 & 24.3 & 1.2 & 0.03 & 97 & 12.3 & $>100$ & $\ldots$ \\
\hline 25 & 26.3 & 26.7 & 24.6 & 1.5 & 0.10 & 115 & 9.3 & $>94$ & $\cdots$ \\
\hline 26 & 26.3 & 28.7 & 25.1 & 0.9 & 0.04 & 49 & 6.5 & $>71$ & $\cdots$ \\
\hline 27 & 25.8 & 25.8 & 24.0 & 2.7 & 0.16 & 180 & 16.3 & 85 & $\ldots$ \\
\hline 28 & 26.1 & 27.0 & 24.8 & 1.4 & 0.12 & 19 & 7.6 & $>77$ & $\ldots$ \\
\hline 29 & 25.5 & 26.0 & 24.4 & 2.0 & 0.29 & 170 & 11.1 & 72 & $\cdots$ \\
\hline
\end{tabular}


Table 4

(Continued)

\begin{tabular}{|c|c|c|c|c|c|c|c|c|c|}
\hline Number $^{a}$ & $\begin{array}{c}i^{\prime \mathrm{b}} \\
\left(\phi 3^{\prime \prime} 0\right)\end{array}$ & $\begin{array}{c}z^{\prime \mathrm{b}} \\
\left(\phi 3^{\prime \prime} \cdot 0\right)\end{array}$ & $\begin{array}{l}\text { NB816 } \\
\left(\phi 3^{\prime \prime} .0\right)\end{array}$ & $\begin{array}{c}\text { FWHM }(\mathrm{NB} 816)^{\mathrm{c}} \\
(\operatorname{arcsec})\end{array}$ & $\epsilon(\mathrm{NB} 816)^{\mathrm{d}}$ & $\begin{array}{c}\text { PA (NB816) } \\
\text { (degree) }\end{array}$ & $\begin{array}{c}L(\operatorname{Ly} \alpha) \\
\left(10^{42} \mathrm{erg} \mathrm{s}^{-1}\right)\end{array}$ & $\begin{array}{c}E W_{0}(\operatorname{Ly} \alpha)^{\mathrm{e}} \\
(\AA)\end{array}$ & $z_{\mathrm{sp}}^{\mathrm{f}}$ \\
\hline 38 & 26.7 & 99.0 & 24.5 & 1.0 & 0.05 & 81 & 10.9 & $>100$ & $\ldots$ \\
\hline 48 & 27.3 & 99.0 & 24.2 & 1.4 & 0.15 & 36 & 14.8 & $>124$ & $\ldots$ \\
\hline 54 & 25.0 & 26.9 & 24.5 & 1.2 & 0.31 & 150 & 10.4 & $>97$ & $\ldots$ \\
\hline 57 & 25.1 & 24.9 & 23.5 & 1.2 & 0.15 & 7 & 22.8 & 51 & $\ldots$ \\
\hline 58 & 28.2 & 99.0 & 24.8 & 1.0 & 0.10 & 46 & 8.5 & $>78$ & $\ldots$ \\
\hline 59 & 27.0 & 26.3 & 24.7 & 1.1 & 0.13 & 145 & 8.3 & 68 & $\ldots$ \\
\hline 61 & 25.3 & 24.3 & 24.0 & 1.4 & 0.17 & 164 & 10.7 & 15 & $\ldots$ \\
\hline 62 & 27.0 & 99.0 & 24.5 & 1.2 & 0.08 & 133 & 11.5 & $>102$ & $\ldots$ \\
\hline 79 & 27.4 & 26.1 & 24.4 & 1.2 & 0.16 & 65 & 11.2 & 79 & 5.686 \\
\hline 80 & 25.7 & 25.5 & 24.3 & 0.9 & 0.06 & 57 & 10.6 & 44 & $\ldots$ \\
\hline 87 & 26.2 & 25.9 & 24.7 & 1.4 & 0.30 & 146 & 7.8 & 46 & $\ldots$ \\
\hline 88 & 27.3 & 99.0 & 24.5 & 1.4 & 0.15 & 38 & 11.4 & $>105$ & $\ldots$ \\
\hline 89 & 26.2 & 29.6 & 24.5 & 1.4 & 0.38 & 173 & 10.8 & $>92$ & $\ldots$ \\
\hline 91 & 25.5 & 29.7 & 24.6 & 1.4 & 0.13 & 166 & 9.9 & $>89$ & $\ldots$ \\
\hline 92 & 27.0 & 99.0 & 24.7 & 1.3 & 0.10 & 133 & 9.5 & $>84$ & $\ldots$ \\
\hline 93 & 28.0 & 99.0 & 24.9 & 1.5 & 0.28 & 61 & 8.8 & $>79$ & $\ldots$ \\
\hline 97 & 26.2 & 25.6 & 24.4 & 1.4 & 0.29 & 34 & 10.2 & 46 & $\ldots$ \\
\hline 100 & 26.6 & 26.3 & 24.8 & 1.4 & 0.24 & 13 & 7.1 & 58 & $\cdots$ \\
\hline 101 & 27.1 & 99.0 & 24.8 & 1.3 & 0.34 & 59 & 10.2 & $>98$ & $\ldots$ \\
\hline 102 & 26.6 & 26.0 & 24.7 & 0.9 & 0.19 & 0 & 7.6 & 50 & $\ldots$ \\
\hline 103 & 28.8 & 26.1 & 24.4 & 1.4 & 0.23 & 171 & 11.2 & 83 & $\cdots$ \\
\hline 106 & 27.1 & 99.0 & 24.7 & 1.6 & 0.20 & 98 & 10.2 & $>100$ & $\ldots$ \\
\hline 108 & 26.0 & 26.1 & 24.2 & 0.9 & 0.15 & 66 & 13.4 & 93 & 5.693 \\
\hline 111 & 25.4 & 24.3 & 23.9 & 1.8 & 0.08 & 45 & 12.6 & 17 & $\ldots$ \\
\hline 112 & 26.7 & 26.0 & 24.6 & 1.5 & 0.03 & 91 & 8.8 & 59 & $\ldots$ \\
\hline 114 & 26.4 & 99.0 & 24.0 & 1.6 & 0.25 & 11 & 18.1 & $>155$ & 5.627 \\
\hline
\end{tabular}

No ACS Image

\begin{tabular}{|c|c|c|c|c|c|c|c|c|c|}
\hline 3 & 26.4 & 26.3 & 24.7 & 1.7 & 0.42 & 165 & 8.2 & 68 & $\ldots$ \\
\hline 8 & 25.7 & 24.8 & 24.2 & 2.0 & 0.33 & 84 & 9.8 & 20 & $\ldots$ \\
\hline 10 & 25.4 & 24.6 & 24.1 & 1.9 & 0.27 & 130 & 11.8 & 22 & $\ldots$ \\
\hline 11 & 24.9 & 24.8 & 24.1 & 2.3 & 0.24 & 107 & 12.2 & 25 & $\ldots$ \\
\hline 12 & 28.1 & 99.0 & 24.7 & 1.0 & 0.13 & 62 & 8.8 & $>69$ & $\ldots$ \\
\hline 17 & 25.4 & 25.2 & 24.2 & 1.3 & 0.25 & 38 & 11.7 & 35 & $\ldots$ \\
\hline 18 & 25.3 & 25.7 & 23.8 & 1.2 & 0.11 & 43 & 19.1 & 91 & $\ldots$ \\
\hline 22 & 26.7 & 99.0 & 24.3 & 1.9 & 0.24 & 119 & 12.8 & $>74$ & $\ldots$ \\
\hline 31 & 25.8 & 26.2 & 23.8 & 1.4 & 0.14 & 36 & 20.3 & 154 & $\ldots$ \\
\hline 32 & 26.6 & 26.1 & 23.9 & 1.3 & 0.17 & 55 & 18.5 & 127 & $\ldots$ \\
\hline 33 & 25.8 & 25.1 & 24.2 & 1.1 & 0.08 & 74 & 11.0 & 30 & 5.639 \\
\hline 36 & 27.2 & 25.9 & 24.5 & 1.5 & 0.15 & 39 & 9.4 & 56 & $\ldots$ \\
\hline 37 & 26.7 & 99.0 & 24.4 & 1.0 & 0.02 & 50 & 12.4 & $>100$ & $\ldots$ \\
\hline 46 & 24.8 & 24.9 & 23.8 & 1.3 & 0.20 & 137 & 16.7 & 39 & $\ldots$ \\
\hline 52 & 25.8 & 25.0 & 24.2 & 1.6 & 0.31 & 34 & 10.7 & 29 & $\ldots$ \\
\hline 53 & 25.1 & 24.9 & 23.5 & 1.1 & 0.10 & 63 & 22.8 & 53 & $\ldots$ \\
\hline 56 & 26.3 & 25.9 & 24.6 & 1.6 & 0.28 & 8 & 8.2 & 47 & $\ldots$ \\
\hline 63 & 26.0 & 25.5 & 24.2 & 1.4 & 0.14 & 80 & 11.8 & 48 & $\ldots$ \\
\hline 64 & 27.1 & 25.8 & 24.5 & 1.5 & 0.11 & 95 & 9.4 & 49 & 5.680 \\
\hline 65 & 26.3 & 26.4 & 24.8 & 1.0 & 0.16 & 5 & 7.4 & 68 & 5.693 \\
\hline 66 & 26.7 & 24.5 & 24.3 & 1.1 & 0.10 & 89 & 7.1 & 11 & 5.656 \\
\hline 67 & 24.7 & 23.7 & 23.3 & 1.6 & 0.12 & 86 & 20.8 & 15 & $\ldots$ \\
\hline 70 & 26.1 & 27.5 & 24.3 & 1.0 & 0.08 & 14 & 12.6 & $>89$ & $\ldots$ \\
\hline 72 & 26.6 & 25.8 & 24.8 & 1.1 & 0.12 & 49 & 6.5 & 35 & $\ldots$ \\
\hline 82 & 26.0 & 25.4 & 24.6 & 1.2 & 0.18 & 48 & 7.7 & 30 & $\ldots$ \\
\hline 90 & 26.5 & 99.0 & 24.7 & 1.4 & 0.15 & 163 & 9.0 & $>78$ & $\ldots$ \\
\hline 94 & 26.3 & 27.1 & 24.8 & 1.3 & 0.30 & 4 & 8.0 & $>64$ & $\ldots$ \\
\hline 109 & 25.9 & 25.3 & 24.3 & 1.1 & 0.26 & 44 & 10.9 & 37 & $\ldots$ \\
\hline 113 & 25.6 & 99.0 & 24.6 & 0.9 & 0.15 & 146 & 10.5 & $>92$ & $\ldots$ \\
\hline 115 & 24.7 & 24.6 & 23.4 & 1.1 & 0.10 & 18 & 26.0 & 45 & $\ldots$ \\
\hline 116 & 26.3 & 25.4 & 24.7 & 1.0 & 0.02 & 27 & 6.3 & 23 & $\ldots$ \\
\hline 117 & 25.1 & 24.8 & 24.1 & 1.7 & 0.17 & 163 & 11.4 & 25 & $\ldots$ \\
\hline
\end{tabular}


Table 4

(Continued)

\begin{tabular}{|c|c|c|c|c|c|c|c|c|c|}
\hline Number $^{a}$ & $\begin{array}{c}i^{\prime \mathrm{b}} \\
\left(\phi 3^{\prime \prime} 0\right)\end{array}$ & $\begin{array}{c}z^{\prime \mathrm{b}} \\
\left(\phi 3^{\prime \prime} 0\right)\end{array}$ & $\begin{array}{l}\text { NB816 } \\
\left(\phi 3^{\prime \prime} 0\right)\end{array}$ & $\begin{array}{c}\text { FWHM (NB816) } \\
(\operatorname{arcsec})\end{array}$ & $\epsilon(\mathrm{NB} 816)^{\mathrm{d}}$ & $\begin{array}{l}\text { PA (NB816) } \\
\text { (degree) }\end{array}$ & $\begin{array}{c}L(\operatorname{Ly} \alpha) \\
\left(10^{42} \mathrm{erg} \mathrm{s}^{-1}\right)\end{array}$ & $\begin{array}{c}E W_{0}(\operatorname{Ly} \alpha)^{\mathrm{e}} \\
(\AA)\end{array}$ & $z_{\mathrm{sp}}{ }^{\mathrm{f}}$ \\
\hline 118 & 25.6 & 25.5 & 23.6 & 1.3 & 0.17 & 53 & 22.8 & 94 & \\
\hline 119 & 25.8 & 25.2 & 24.5 & 1.7 & 0.36 & 41 & 8.2 & 25 & $\cdots$ \\
\hline
\end{tabular}

Notes.

${ }^{a}$ The LAE IDs given in Murayama et al. (2007).

b AB magnitude. An entry of " 99.0 " indicates that no excess flux was measured.

${ }^{c}$ Spatial sizes measured on NB816 images.

${ }^{\mathrm{d}}$ Ellipticity measured on NB816 images.

${ }^{\mathrm{e}}$ Lower limits represent $1 \sigma$ significance.

${ }^{\mathrm{f}}$ Spectroscopic redshift.

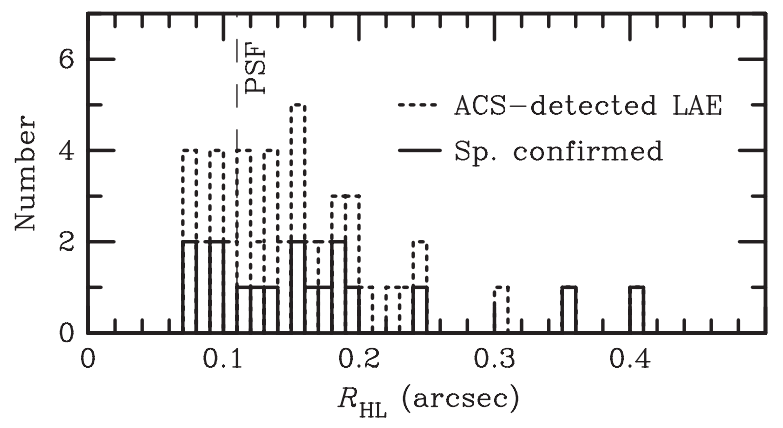

Figure 4. Distribution of the half-light radius in the ACS images, $R_{\mathrm{HL}}$, for the 47 LAEs detected with ACS by a dotted histogram. LAEs with spectroscopic confirmation are shown by a solid histogram. The PSF size $(0.11 \mathrm{arcsec})$ derived from stars is shown by the dashed line.

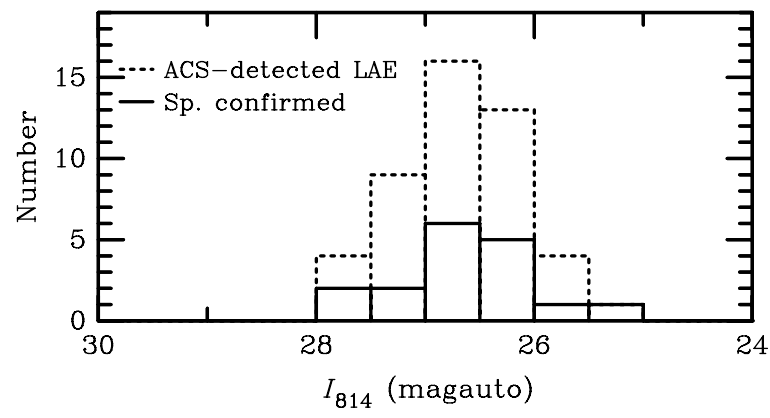

Figure 5. Frequency distribution of the ACS magnitude $I_{814}$ by a dotted histogram. LAEs confirmed by our follow-up spectroscopy are shown as a solid histogram.

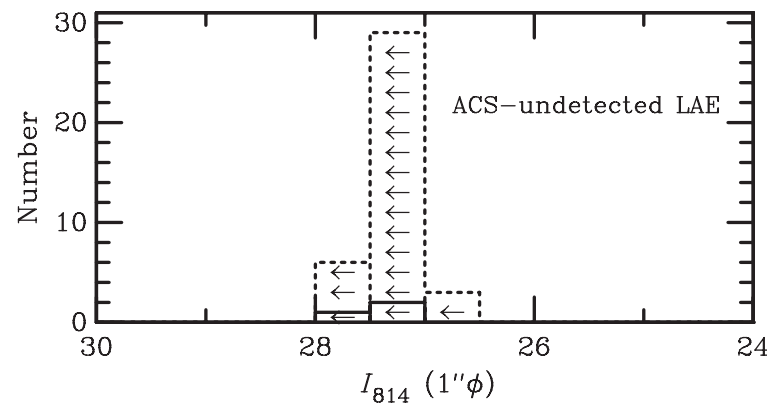

Figure 6. Frequency distribution of the ACS magnitude $I_{814}$ for the ACSundetected LAEs (dotted histogram); $3 \sigma$ upper limits are given for each LAE. LAEs confirmed by our follow-up spectroscopy are shown by a solid histogram.

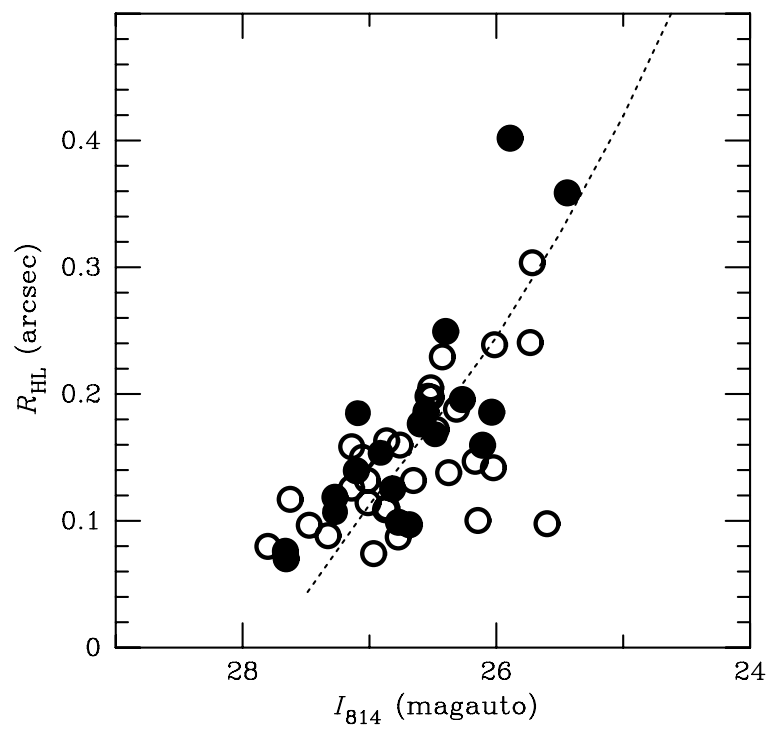

Figure 7. Diagram between half-light radius $R_{\mathrm{HL}}$ and the ACS magnitude $I_{814}$. The LAEs confirmed by our follow-up spectroscopy are shown by filled circle. For double-component LAEs, each component is plotted. The 50\% detection completeness for exponential disk objects estimated by Monte Carlo simulation is indicated by the dashed curve.

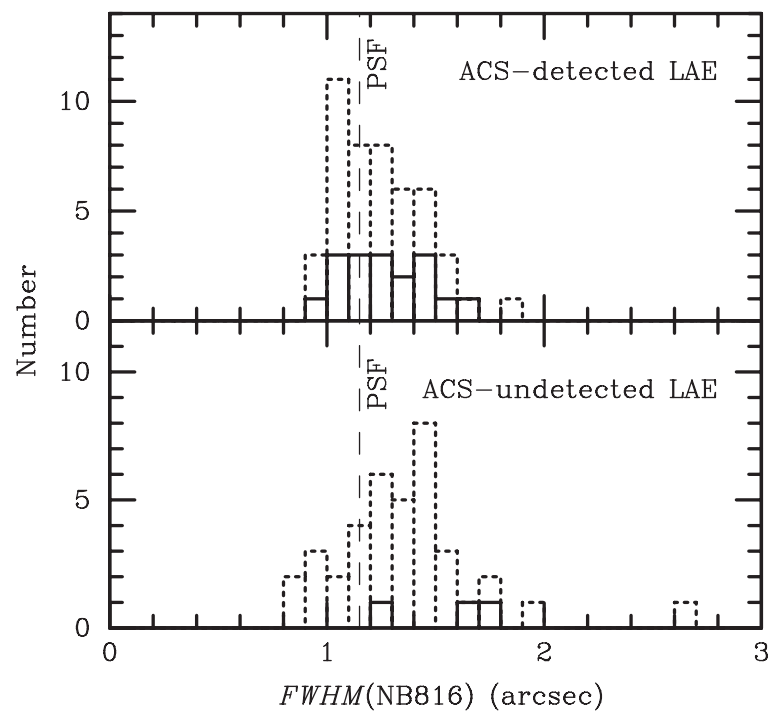

Figure 8. Frequency distributions of size in the narrowband filter NB816 FWHM (NB816) for the ACS-detected and ACS-undetected LAEs by dotted histograms. LAEs confirmed by our follow-up spectroscopy are shown by solid histograms. 


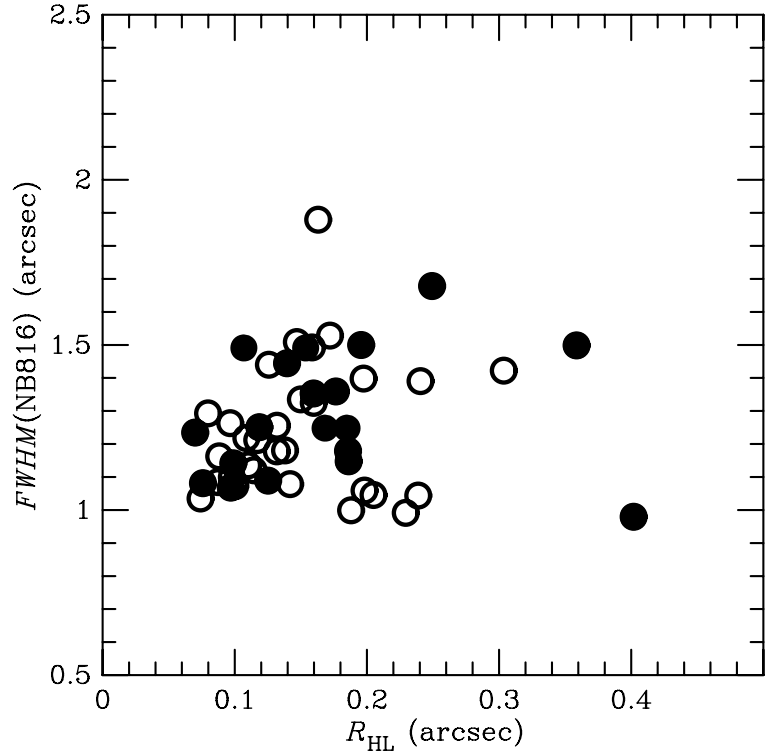

Figure 9. Diagram between size in the narrowband filter NB816, FWHM (NB816), and the half-light radius in the ACS image, $R_{\mathrm{HL}}$. LAEs confirmed by our follow-up spectroscopy are shown by filled circles. For double-component LAEs, $R_{\mathrm{HL}}$ is plotted for each component, while the same FWHM (NB816) is adopted for the two components as they are not resolved in the NB816 images.
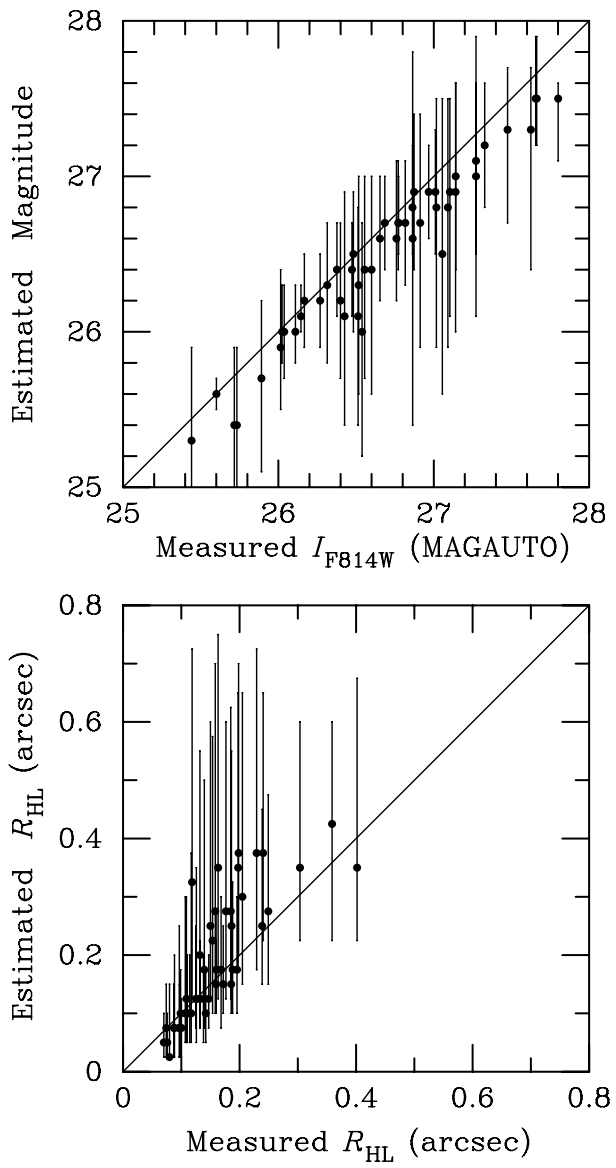

Figure 10. Results of the Monte Carlo simulation: the relation between estimated total magnitudes by the simulation and measured magnitudes of our LAE sample (upper panel) and the relation between estimated half-light radii by the simulation and measured half-light radii (upper panel).

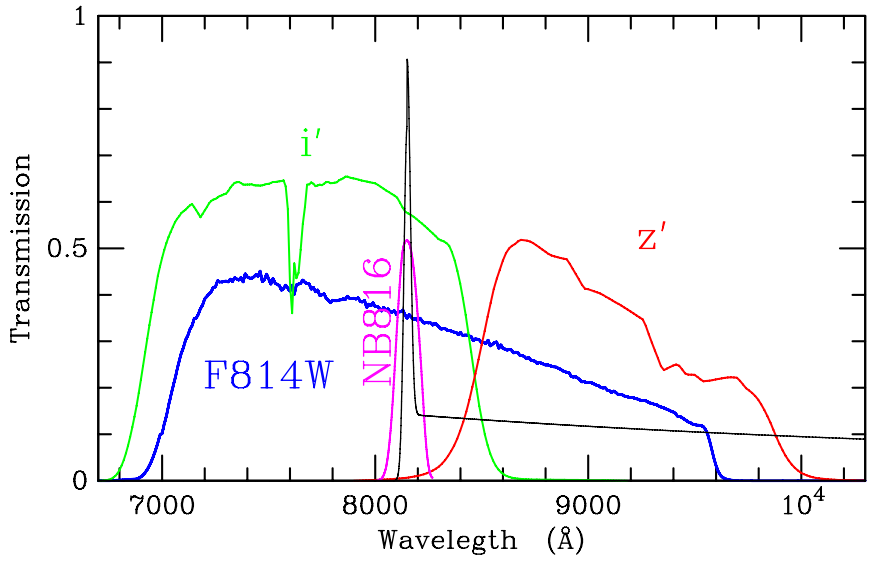

Figure 11. Transmission curves for the filters used in our analysis: F814W (blue curve) for ACS/HST and $i^{\prime}$ (green curve), NB816 (magenta curve), and $z^{\prime}$ (red curve) for Suprime-Cam/Subaru. The CCD sensitivity is taken into account for each filter transmission curve. Model spectrum (black curve) of a LAE at $z \approx 5.7$ with a rest-frame $\mathrm{EW}$ of $30 \AA$ is also plotted.

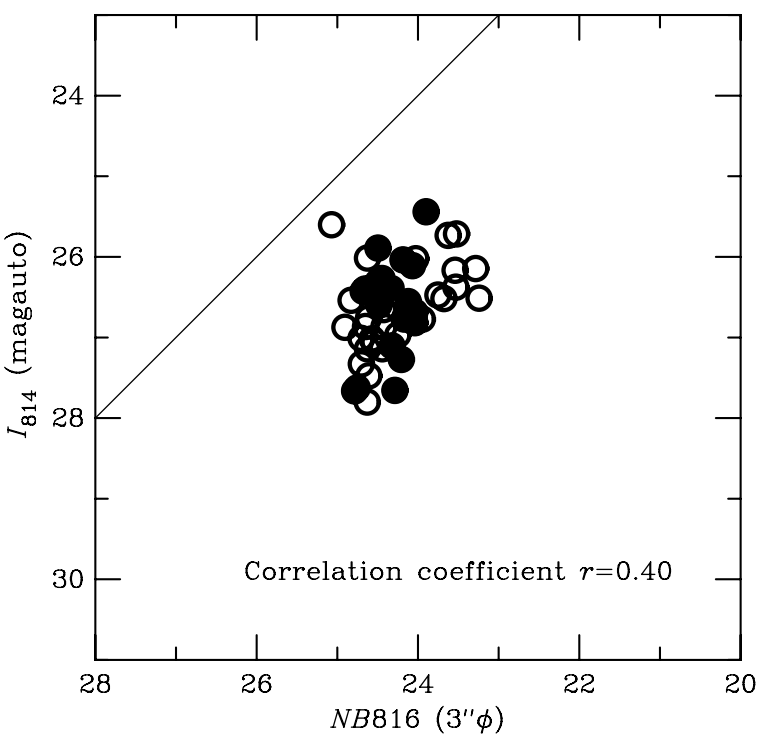

Figure 12. Diagram between $I_{814}$ and NB816. LAEs confirmed by our followup spectroscopy are shown by filled circles. For the double-component LAEs, $I_{814}$ is the total magnitude of the two components. Their NB816 magnitudes are measured with a $3^{\prime \prime}$ aperture that included the two components.

Since the sizes in NB816 of the ACS-undetected LAEs are systematically larger than those of the ACS-detected LAEs (Figure 8), it is suggested that a faint compact UV continuum source with a widely extended bright Ly $\alpha$ nebula explains for the undetection in $I_{814}$ for a part of the ACS-undetected LAEs. However, at least for the 16 ACS-undetected LAEs with $I_{814}\left(z^{\prime}\right)$ $\leqslant 26.5$, their rest EW $(\operatorname{Ly} \alpha)$ is comparable with that of ACSdetected LAEs so that they would be detected in ACS if they had a compact $\left(R_{\mathrm{HL}}<0.15\right.$ arcsec) UV continuum source. Therefore, they probably have an extended UV continuum source.

\section{DISCUSSION}

\subsection{ACS Size and Star Formation Properties}

In the previous section, we have demonstrated that our ACS imaging with $\mathrm{F} 814 \mathrm{~W}$ does not probe $\mathrm{Ly} \alpha$ emission but UV continuum from massive stars in the LAEs. Since all LAEs detected in the ACS imaging are spatially small (i.e., $<0.4$ arcsec or $<2.5 \mathrm{kpc}$ ), their star-forming regions are considered 

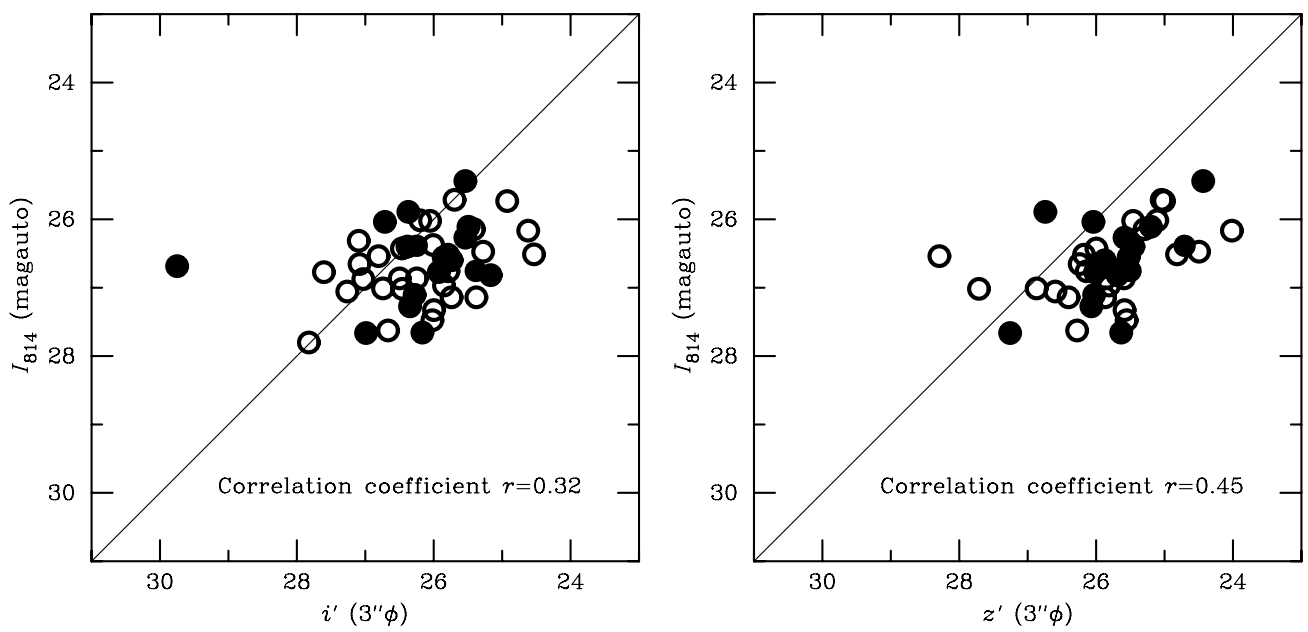

Figure 13. Diagrams between $I_{814}$ and $i^{\prime}$ (left), $z^{\prime}$ (right). LAEs confirmed by our follow-up spectroscopy are shown by filled circles. For the double-component LAEs, $I_{814}$ is the total magnitude of the two components. Their $i^{\prime}$ and $z^{\prime}$ magnitudes are measured with a $3^{\prime \prime}$ aperture that included the two components.

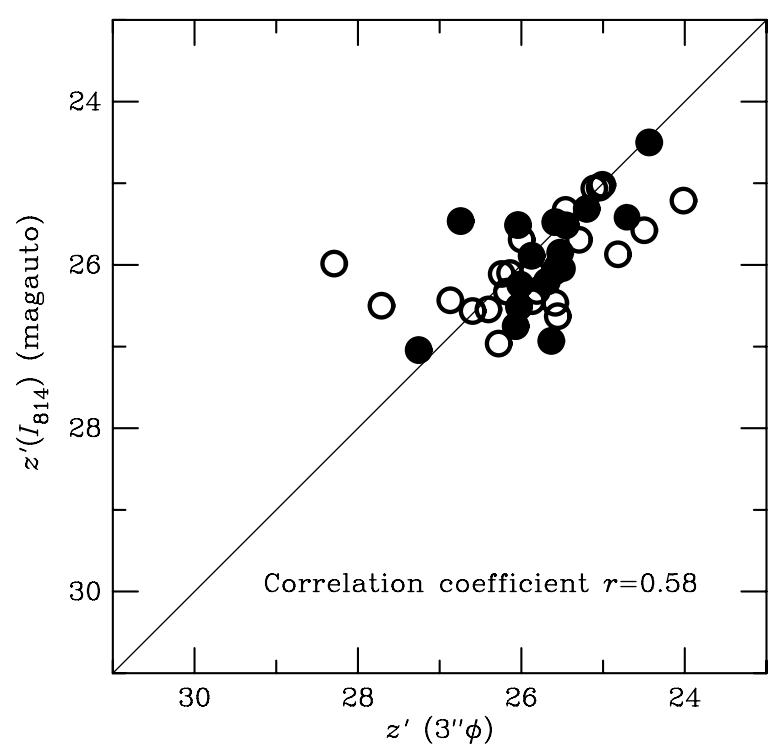

Figure 14. Diagrams between the corrected $z\left(I_{814}\right)$ and $z^{\prime}$. LAEs confirmed by our follow-up spectroscopy are shown by filled circles. For $z\left(I_{814}\right)$ of the double-component LAEs, their total magnitudes of the two components are used. Their Subaru $z^{\prime}$ magnitudes are measured with a $3^{\prime \prime}$ aperture that included both components.

to be physically compact. The sizes of high- $z$ galaxies provide important information on the growth of the luminous parts of galaxies embedded in dark matter haloes (e.g., Dalcanton et al. 1997; Mo et al. 1998; Ferguson et al. 2004; Bouwens et al. 2006).

Here, we investigate how the size of the UV continuum is related to the global star formation properties in the LAEs. In Figure 15, we show the diagram between $R_{\mathrm{HL}}$ and $L(\mathrm{Ly} \alpha)$. In Figure 16, we present the diagram between $R_{\mathrm{HL}}$ and the restframe EW $(\operatorname{Ly} \alpha)$. We find no correlation in these two diagrams. These results suggest that the size of UV continuum regions is not directly related to the star-forming activity traced by Ly $\alpha$ emission in our LAEs. However, one would naturally have expected positive correlations in these diagrams. Therefore, the little correlation implies the following three possibilities: (1) most star-forming regions have lower surface brightness than our detection limit; (2) most star-forming regions are hidden by dusty clouds; or (3) the UV continuum probes star-forming regions with a large age spread while Ly $\alpha$ only probes youngest

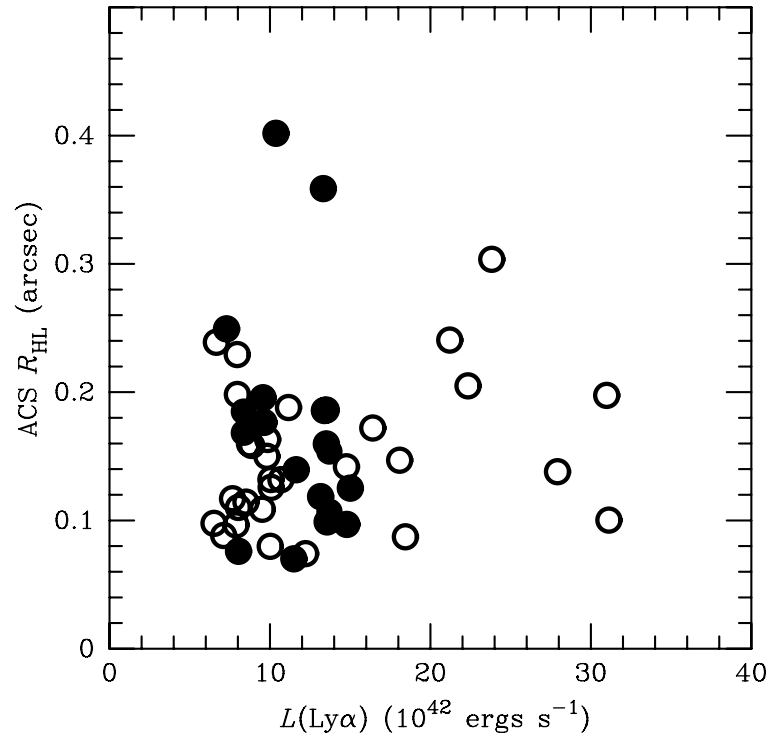

Figure 15. Diagrams between $R_{\mathrm{HL}}$ and $L(\mathrm{Ly} \alpha)$. LAEs confirmed by our followup spectroscopy are shown by filled circles. For double-component LAEs, $R_{\mathrm{HL}}$ is plotted for each component while the same $L(\operatorname{Ly} \alpha)$ is adopted for the two components, because they are not resolved in the ground-based images.

star-forming regions that might not spatially overlap with older star-forming regions. Radio and millimeter stacking analysis for our LAE sample rules out large dust content (Carilli et al. 2007). Since a number of observations also suggest that LAEs at high redshift tend to have little dust content (Lai et al. 2007; Finkelstein et al. 2008, 2009a, 2009b; see, however, Chary et al. 2005), the second possibility appears to be unlikely. Currently, however, we have no firm answer on this issue. In the future, we need deeper F814 imaging of our LAEs. Also, sensitive restframe, mid- and far-infrared imaging will be necessary to solve this problem.

\subsection{Structural Properties of the LAEs Detected in Our ACS Imaging}

As we have shown, our ACS imaging with F814W probes UV continuum from massive stars in the LAEs and the majority of our LAEs show extended morphology in $I_{814}$. It is of great interest to analyze not only their sizes but also their surface 


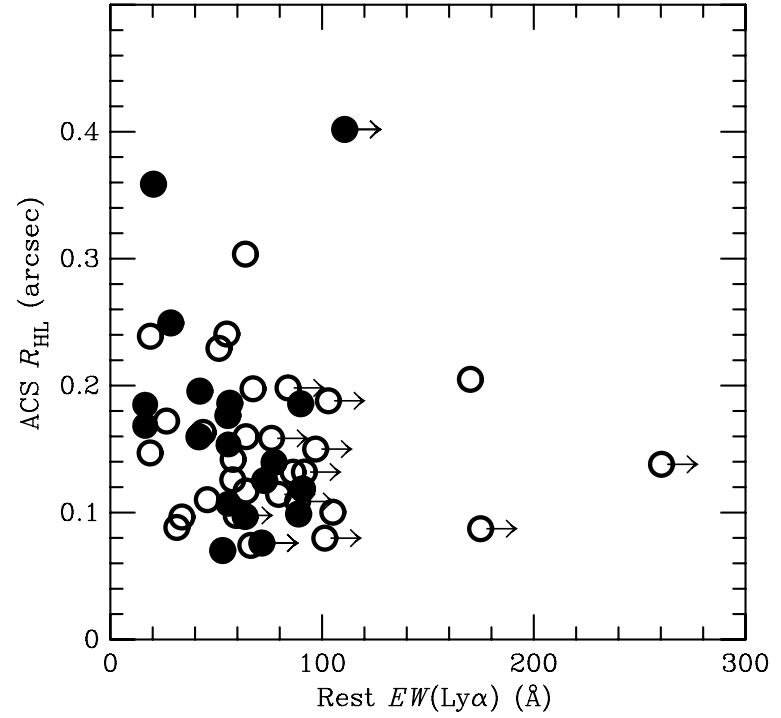

Figure 16. Diagrams between $R_{\mathrm{HL}}$ and EW (Ly $\left.\alpha\right)$. LAEs confirmed by our follow-up spectroscopy are shown by filled circles. LAEs confirmed by our follow-up spectroscopy are shown by filled circles. For double-component LAEs $R_{\mathrm{HL}}$ is plotted for each component while the same $\mathrm{EW}(\operatorname{Ly} \alpha)$ is adopted for the two components, because they are not resolved in the ground-based images.

brightness profiles. Unfortunately, our ACS images are not deep enough to perform such an analysis for each LAE individually. Therefore, we alternatively adopt the image stacking method to obtain mean properties for our LAEs at $z \approx 5.7$.

First, we rotated the major axis to align in the $x$-axis for each LAE image. In this procedure, we used the position angles measured by SExtractor. We also constructed second rotated images in 180 degree from the first rotated (majoraxis aligned) images. Next, we generated a composite image by coadding counts of both the first and second rotated images of LAEs. In this analysis, we did not use the double-component LAEs, No. 60 and No. 110. We also excluded No. 73 and No. 81 because foreground galaxies are very close to these LAE sources. Therefore, we used the remaining 43 LAEs for the stacking analysis.

Finally, we measured structural parameters on the resultant image by modeling one-dimensional surface brightness profile along the major axis. The PSF image required for convolving the model light distribution was derived by combining stellar images near the LAEs. Assuming a Sersic function, we obtained the PSF-deconvolved effective radius of $R_{\mathrm{HL}}=0.13_{-0.01}^{+0.03} \operatorname{arcsec}$ $(0.76 \mathrm{kpc})$ and the Sersic index $(n)$ of $n=0.7_{-0.3}^{+0.3}$. In Figure 17, we show the composite images, light profiles of the composite image along the major axis, and the model light profile. Note that the observed effective radius of stars are typically 0.11 arcsec, and thus the composite LAE image is only slightly resolved. The derived parameters may contain larger uncertainties than the nominal errors estimated from the residuals. However, the derived small Sersic index suggests that our LAEs may have on average irregular or disk-like morphologies rather than spheroidal structures.

Ravindranath et al. (2006) found that $40 \%$ of LBGs at $z>2.5$ have exponential profiles, $30 \%$ of LBGs have $r^{1 / 4}$-like profiles, and $30 \%$ of LBGs have multiple cores. In contrast, our analysis indicates that spheroidal structures are rare in our LAE sample at $z \approx 5.7$. Note that clumpy- or chain-like structures are often seen in galaxies at $z=1-5$ in the UDF sample (e.g., Elmegreen et al. 2007). Since our stacking analysis smears out such fine

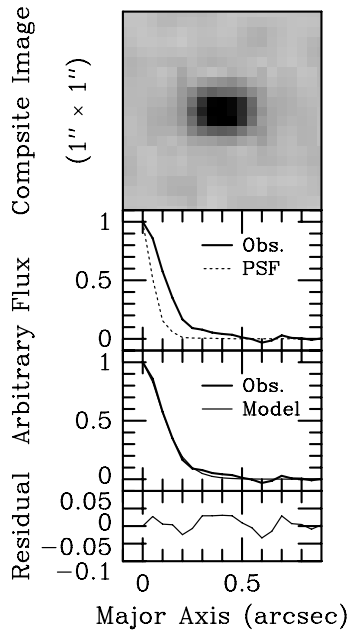

Figure 17. Composite (stacked) ACS image of all LAEs. The surface brightness profiles (thick lines), the model profile (the thin line in the third panel), and residuals (the thin line in the bottom panel) are plotted. The light profile of the PSF (dashed line) derived from stars in the ACS image is also indicated for comparison.

structures, we cannot rule out that our LAE sample includes LAEs with irregular morphologies in the hierarchical build-up process (e.g., Hathi et al. 2008).

\subsection{Relationships Between LAEs and LBGS}

Since LAEs and LBGs are two major populations of starforming galaxies at high redshift, it has been often discussed what their physical and evolutionary relations are. LAEs are selected by the narrowband imaging technique that probes strong Ly $\alpha$ line emission (see for reviews, Taniguchi et al. 2005; Taniguchi 2008). This technique does not require that the UV continuum emission is strong enough to be detected in optical broadband imaging. The larger EW (Ly $\alpha)$ suggest that they tend be younger in context of the elapsed time from the onset of star formation activity (e.g., Rhoads \& Malhotra 2001; Malhotra \& Rhoads 2002; Nagao et al. 2007). Therefore, it is expected that LAEs tend to be young less massive star-forming galaxies. On the other hand, LBGs are selected as so-called dropout objects in broadband images (e.g., Steidel et al. 1999). This technique requires that the UV continuum is strong enough to be detected. However, the selection of dropouts is not affected by the strength of the Ly $\alpha$ line in principle (e.g., Steidel et al. 2000; Shapley et al. 2003). Therefore, LBGs tend to be more massive and relatively older than LAEs.

In fact, several studies of LAEs and/or LBGs support the above difference between the two populations at $z \sim 3-5$ as follows. For example, LAEs tend to have bluer UV continua than LBGs and this property cannot be explained only with a difference in reddening by dust (e.g., Gronwall et al. 2007; Ouchi et al. 2008). However, the number fraction of LAE/LBG tends to increase with increasing redshift; i.e., from several to $10 \%$ at $z \sim 3$ to $\sim 30 \%$ at $z \sim 6-7$ (e.g., Shimasaku et al. 2006; Dow-Hygelund et al. 2007; Ouchi et al. 2008; R. Sumiya et al. 2009 , in preparation). Since, at $z \sim 6$, the elapsed time from the Big Bang is at more 1 Gyr. LBGs and LAEs may tend to share nearly the same physical properties although their Ly $\alpha$ emission luminosities are different on the average. Therefore, it seems important to compare observational properties between LBGs and LAEs at $z \sim 6$ in a more systematic way (e.g., DowHygelund et al. 2007; Pentericci et al. 2007). Motivated by this, we discuss the size-mass relation for both LBGs and LAEs 
Table 5

Symbols and References for Figure 18

\begin{tabular}{|c|c|c|}
\hline Symbol & Description & References \\
\hline Large red filled circle & COSMOS LAEs at $z \approx 5.7$ & This study \\
\hline Small black filled circle & $i$-band dropout galaxies in UDF, UDF-P, GOODS-N, and GOODS-S & Bouwens et al. (2006) \\
\hline Small red filled circle & Spectroscopic confirmed LAEs among $i$-band dropout galaxies in GOODS-S & Bouwens et al. (2006) \\
\hline Small blue filled circle & Spectroscopic confirmed LAEs among $i$-band dropout galaxies in GOODS-S & $\begin{array}{l}\text { ESO GOODS-S database }^{\mathrm{a}} \\
\text { Bouwens et al. (2006) } \\
\text { ESO GOODS-S database }^{\mathrm{a}}\end{array}$ \\
\hline Red plus & LAE at $z=5.7$ & Bunker et al. (2003) \\
\hline Black asterisk & $i$-band dropout galaxies in UDF & Bunker et al. (2004) \\
\hline Red open circle & LAEs at $z \sim 5.8$ & Stanway et al. (2004a) \\
\hline Red cross & LAEs in GOODS-N & Stanway et al. (2004b) \\
\hline Blue open square & Non-emission-line galaxies at $z \sim 6$ & Dow-Hygelund et al. (2007) \\
\hline Red open square & Spectroscopic confirmed LAEs at $z \sim 6$ & Dow-Hygelund et al. (2007) \\
\hline
\end{tabular}

Note.

${ }^{\text {a }}$ Spectroscopic properties for the sample from Bouwens et al. (2006) were taken from the ESO GOODS/VIRMOS spectroscopic database at http://www.eso.org/science/goods/.

using our own data presented here together with all available data from the literature.

Recently, Dow-Hygelund et al. (2007) made a spectroscopic study of 22 LBGs around $z \sim 6$ selected from the samples in three different sky areas and they identified six LBGs with Ly $\alpha$ emission (i.e., LAEs) at $z=5.5-6.1$. They investigated the sizemagnitude relation for both LAEs and LBGs compiled from the literature as well as their own data. They defined the size as the half-light radius $\left(R_{\mathrm{HL}}\right)$ and the magnitude is ACS $z_{850}$ magnitude (see Figure 13 in their paper). They found that the LAEs tend to be more compact than the LBGs at $z \sim 6$, suggesting that the LAEs are younger than the LBGs. This interpretation may be supported by other observational differences between LAEs and LBGs; LAEs tend to be less massive (e.g., Overzier et al. 2006; Gawiser et al. 2006; Pentericci et al. 2007).

In order to increase the sample size for an analysis of the sizemagnitude relation compared to Dow-Hygelund et al. (2007), we have also compiled available data from the literature for $z \sim 6$. Our data compilations are summarized in Table 5. Since our ACS magnitude is not $z_{850}$ but $I_{814}$, we have to convert our $I_{814}$ magnitude to $z_{850}$. In this procedure, we assume that the flux of the rest-frame continuum at $\lambda<912 \AA$ is zero, while that at $\lambda \geqslant 912 \AA$ is constant. We also calculate the contribution of the $\operatorname{Ly} \alpha$ flux by using the rest-frame EW (Ly $\alpha)$. In this way, we derive the following equation for conversion:

$$
z_{850}=I_{814}+2.5 \log \left(0.364+2.088 \times 10^{-3} E W_{0}\right) .
$$

In Figure 18, we show our own results together with the compiled data for $z \sim 6$. Our new data presented in the right panel add more information on this size-magnitude diagram with respect to that presented by Dow-Hygelund et al. (2007). We find that there is little difference between the two populations, LAEs and LBGs at $z \sim 6$, although there many faint LBGs taken from the very deep ACS imaging of LBGs made by Bouwens et al. (2006) and Bunker et al. (2003) are located at $z_{850}<29.5$.

\section{CONCLUSIONS}

We have derived the detailed morphological properties of Ly $\alpha$ emitters (LAEs) at $z \approx 5.7$ in the COSMOS field, based on the HST ACS imaging in the F814W filter. Our main results and conclusions are summarized below.

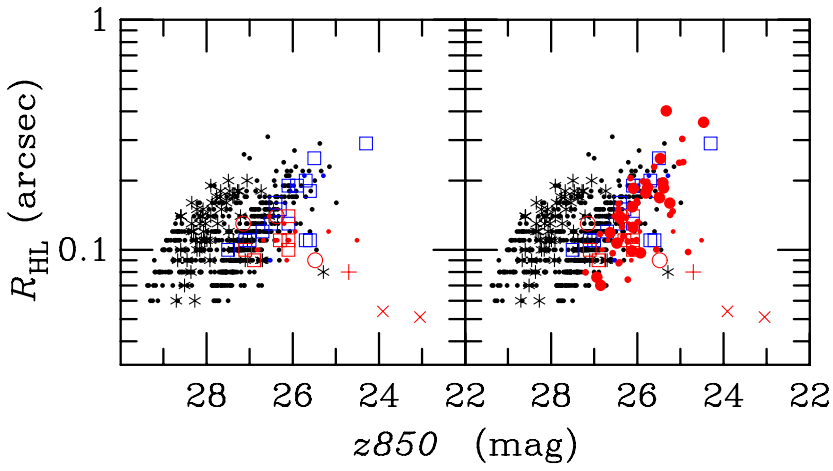

Figure 18. Diagrams between $R_{\mathrm{HL}}$ and $z_{850}$ for $z \sim 6$. The left panel is the same as the right panel except that our data are excluded for clarity. Spectroscopically confirmed LAEs are shown by the red symbols, and spectroscopically confirmed LBGs without Ly $\alpha$ emission are shown by the blue symbols. Our LAEs are shown by large red filled circles. For the double-component LAEs, each component is plotted. See Table 5 for explanation of the remaining symbols.

1. Among the 119 LAEs at $z=5.7$ identified in the $H S T$ COSMOS field (M07), 85 LAEs are imaged with ACS/ F814W. Of those, 47 LAEs are detected in our ACS imaging while the remaining 38 ones are not detected.

2. All LAEs detected in ACS have small spatial sizes $\left(R_{\mathrm{HL}} \lesssim\right.$ $0.4 \operatorname{arcsec}$ ). However, nearly half of them show a spatially extended morphology with effective radii larger than 0.15 $\operatorname{arcsec}(0.93 \mathrm{kpc})$, which is larger than the PSF size $\left(R_{\mathrm{HL}}=0.11\right.$ arcsec $)$.

3. Among the 38 ACS-undetected LAEs, 16 LAEs may be spatially extended $\left(R_{\mathrm{HL}} \geqslant 0.15\right.$ arcsec $)$ as estimated from their $z^{\prime}$ and NB816 magnitudes.

4. Comparing the ACS data with our Subaru NB816, $i^{\prime}$, and $z^{\prime}$ data, we find that the ACS/F814 imaging probes not Ly $\alpha$ line emission but UV continuum arising from wavelengths longer than $1216 \AA$.

5. We find a tendency that LAEs with a larger UV continuum source have a larger Ly $\alpha$ size as probed by our Subaru NB816 imaging. Since the ACS-undetected LAEs have systematically larger FWHM (NB816) than ACS-detected LAEs, they may have a large UV continuum size, and thus their surface brightness in $I_{814}$ falls below our detection limit.

6. UV continuum sizes of LAEs are not directly related to star formation properties such as Ly $\alpha$ luminosity and Ly $\alpha$ equivalent widths. 
We thank both the Subaru and HST staff for their invaluable help, and all members of the COSMOS team. We also thank the anonymous referee for his/her useful comments. This work was in part financially supported by JSPS (15340059 and 17253001).

\section{REFERENCES}

Ajiki, M., et al. 2003, AJ, 126, 2091

Bertin, E., \& Arnouts, S. 1996, A\&AS, 117, 393

Bouwens, R. J., \& Illingworth, G. D. 2006, Nature, 443, 189

Bouwens, R. J., et al. 2006, ApJ, 653, 53

Bouwens, R. J., et al. 2008, ApJ, 686, 230

Bouwens, R. J., et al. 2009, ApJ, 690, 1764

Bradley, L. D., et al. 2008, ApJ, 678, 647

Bunker, A. J., et al. 2003, MNRAS, 342, L47

Bunker, A. J., et al. 2004, MNRAS, 355, 374

Capak, P., et al. 2007, ApJS, 172, 99

Carilli, C. L., et al. 2007, ApJS, 172, 518

Chary, R.-R., Stein, D., \& Eisenhardt, P. 2005, ApJ, 635, L5

Dalcanton, J. J., Spergel, D. N., \& Summers, F. J. 1997, ApJ, 482, 659

Dow-Hygelund, C. C., et al. 2007, ApJ, 660, 47

Elmegreen, D. N., et al. 2007, ApJ, 658, 763

Ferguson, H. C., et al. 2004, ApJ, 600, L107

Finkelstein, S. L., Malhotra, S., Rhoads, J. E., Hathi, N. P., \& Pizkal, N. 2009a, MNRAS, 393, 1174

Finkelstein, S. L., Roads, J. E., Malhotra, S., \& Grogin, N. 2009b, ApJ, 691, 465

Finkelstein, S. L., et al. 2008, ApJ, 678, 655

Fynbo, J. U., et al. 1999, MNRAS, 305, 849

Gawiser, E., et al. 2006, ApJ, 642, L13

Gronwall, C., et al. 2007, ApJ, 667, 79

Hathi, N. P., Jansen, R. A., Windhorst, R. A., Cohen, S. H., Keel, W. C., Corbin, M. R., \& Ryan, R. E., Jr. 2008, AJ, 135, 156

Hu, E. M., Cowie, L. L., Capak, P., McMahon, R. G., Hayashino, T., \& Komiyama, Y. 2004, AJ, 127, 563
Iye, M., et al. 2004, PASJ, 56, 381

Kaifu, N., et al. 2000, PASJ, 52, 1

Koekemoer, A., et al. 2007, ApJS, 172, 196

Lai, K., et al. 2007, ApJ, 655, 704

Malhotra, S., \& Rhoads, J. E. 2002, ApJ, 565, L71

Matsuda, Y., et al. 2004, AJ, 128, 569

Miyazaki, S., et al. 2002, PASJ, 54, 833

Murayama, T., et al. 2007, ApJS, 172, 523

Mo, H. J., Mao, S., \& White, S. D. M. 1998, MNRAS, 295, 319

Nagao, T., et al. 2007, A\&A, 468, 877

Ouchi, M., et al. 2005, ApJ, 620, L1

Ouchi, M., et al. 2008, ApJS, 176, 301

Overzier, R. A., et al. 2006, ApJ, 637, 58

Overzier, R. A., et al. 2008, ApJ, 673, 143

Pentericci, L., et al. 2007, A\&A, 471, 433

Pizkal, N., et al. 2007, ApJ, 667, 49

Ravindranath, S., et al. 2006, ApJ, 652, 963

Rhoads, J. E., \& Malhotra, S. 2001, ApJ, 563, L5

Rhoads, J. E., et al. 2005, ApJ, 621, 582

Saito, T., et al. 2006, ApJ, 648, 54

Scoville, N. Z., et al. 2007a, ApJS, 172, 1

Scoville, N. Z., et al. 2007b, ApJS, 172, 38

Shimasaku, K., et al. 2006, PASJ, 58, 313

Shapley, A., Steidel, C. C., Pettini, M., \& Adelberger, K. L. 2003, ApJ, 588, 65

Springel, V., et al. 2005, Nature, 435, 629

Stanway, E. R., et al. 2004a, ApJ, 604, L13

Stanway, E. R., et al. 2004b, ApJ, 604, 704

Steidel, C. C., Adelberger, K. L., Giavalisco, M., Dickinson, M., \& Pettini, M. 1999, ApJ, 519, 1

Steidel, C. C., Adelberger, K. L., Shapley, A. E., Pettini, M., Dickinson, M., \& Giavalisco, M. 2000, ApJ, 532, 170

Taniguchi, Y. 2008, in IAU Symp. 250, Massive Stars as Cosmic Engine, ed. F Bresolin, P. Crowther, \& J. Puls (Cambridge: Cambridge Univ. Press), 429 Taniguchi, Y., et al. 2005, PASJ, 57, 165

Taniguchi, Y., et al. 2007, ApJS, 172, 9

Venemans, B. P., et al. 2005, A\&A, 431, 793 